Document downloaded from:

http://hdl.handle.net/10251/141530

This paper must be cited as:

Gutierrez Garcia, J.; Rodríguez López, J.; Romaguera Bonilla, S. (2018). On fuzzy uniformities induced by a fuzzy metric space. Fuzzy Sets and Systems. 330:52-78. https://doi.org/10.1016/j.fss.2017.05.001

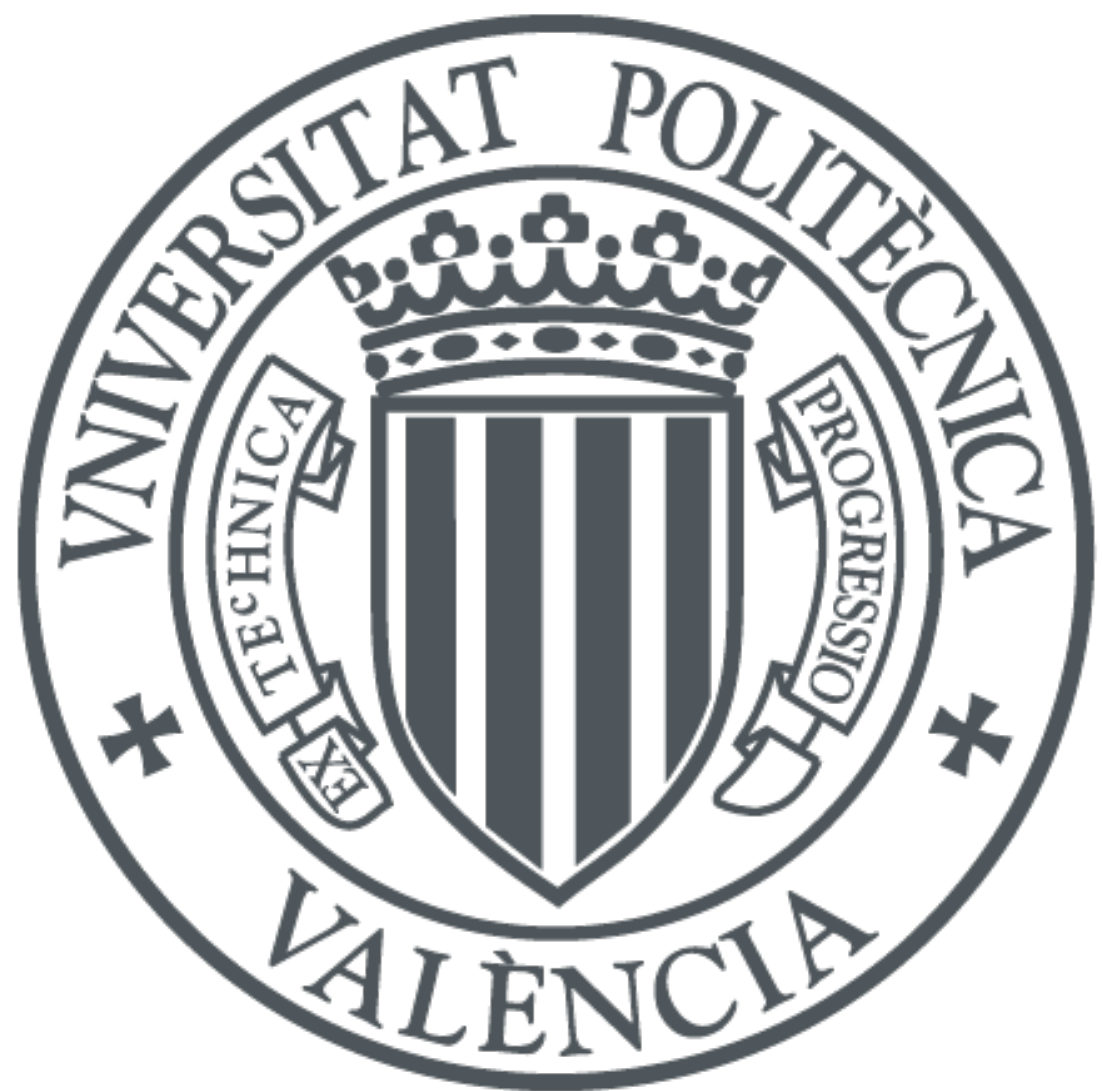

The final publication is available at

https://doi.org/10.1016/j.fss.2017.05.001

Copyright Elsevier

Additional Information 


\title{
ON FUZZY UNIFORMITIES INDUCED BY A FUZZY METRIC SPACE
}

\author{
JAVIER GUTIÉRREZ GARCÍA, JESÚS RODRÍGUEZ-LÓPEZ AND \\ SALVADOR ROMAGUERA
}

\begin{abstract}
Different types of fuzzy uniformities have been introduced in the literature standing out the notions due to Hutton, Höhle and Lowen. The main purpose of this paper is to study several methods to endow a fuzzy metric space $(X, M, *)$, in the sense of George and Veeramani, with a probabilistic uniformity and with a Hutton $[0,1]$ (-quasi)-uniformity. We will show the functorial behaviour of these constructions as well as its relation with respect to Lowen's functors and Katsaras's functors, which establish a relationship between the categories of probabilistic uniformities and Hutton [0,1](-quasi)-uniformities with the category of classical uniformities respectively. Furthermore, we also study the fuzzy topologies associated with these fuzzy uniformities.
\end{abstract}

2010 Mathematics Subject Classification: 54A40; 54E15; 54E70; 54E35.

Keywords and Phrases: fuzzy metric space; probabilistic uniformity; Lowen uniformity; Hutton [0,1]-uniformity; fuzzifying uniformity; [0,1]-topology.

\section{InTRODUCTION}

The problem of finding an appropriate notion of uniformity in the fuzzy context goes back to Hutton [20] (Hutton L-uniformity), Höhle [16] (probabilistic uniformity) and Lowen [27] (Lowen uniformity). These different approaches to the concept of fuzzy uniformity give rise to different techniques and results which has been studied by several authors $[1,21,32,42]$, etc. It is worth mentioning that Lowen [27] introduced two adjoint functors which allow to construct a classical uniformity from a Lowen uniformity and vice versa. On his behalf, Katsaras [22] did the same for Hutton $L$-uniformities. Furthermore, Höhle [16, Remark 2.2] showed how to construct a Hutton $L$-uniformity from a probabilistic uniformity.

Although these are the most well-known approaches to the notion of fuzzy uniformity, others can be found in the literature [2, 15, 39], etc. In [42], Zhang analyzed the relationship between several concepts of fuzzy uniformities.

Another topological structure which has deserved a lot of attention in the fuzzy context is that of a metric. The problem of constructing a satisfactory theory of fuzzy metric spaces has been investigated by several authors from different point of view starting with the statistical metric spaces of Menger [31], which where studied by Schweizer and Sklar [36] under the name of probabilistic metric spaces. Strictly speaking in the context of fuzzy theory, one of the usual concepts of fuzzy metric is that due to George and Veeramani [6], which was in turn motivated by

Date: March 31, 2017.

The first named author acknowledges the support of the grants MTM2015-63608-P (MINECO/FEDER, UE) and IT974-16 (Basque Government). The second named author is supported by the grant MTM2015-64373-P (MINECO/FEDER, UE). 
the definition given by Kramosil and Michalek [24] who adapted the concept of probabilistic metric to the fuzzy setting.

Then it appears in a natural way the problem of studying the relationship between the different concepts of fuzzy metrics and fuzzy uniformities. The first results in this direction are due to Höhle, who showed how a probabilistic pseudometric can generate a probabilistic uniformity [16] and a Lowen uniformity [19]. He also characterized those Lowen uniformities which are probabilistic metrizable [19]. The same problem was also studied by Katsaras [23] for Lowen uniformities with respect to the $t$-norm $\wedge$. He also proved explicitly that these uniformities can be generated by a gauge of probabilistic pseudometrics (see also [42]). On their behalf, Gutiérrez García, Romaguera and Sanchis [15] associated to a fuzzy metric space a fuzzy uniformity by means of a family of fuzzy pseudometrics satisfying certain properties.

On the other hand, in [12] the authors associated to each fuzzy metric space a Hutton quasi-uniformity (see Proposition 5.8) and this association factorizes, via a Katsaras' functor, the association of uniform spaces to fuzzy metric spaces. Furthermore, Yue and Shi [40] constructed a fuzzifying uniformity by means of a fuzzy pseudometric (see also [30]). Recently, Yue and Fang [41] have constructed an $I$-uniformity in a fuzzy metric space in the sense of Kramosil and Michalek. This I-uniformity is generated by the Hutton quasi-uniformity of [12] as well as by other different Hutton quasi-uniformity constructed in [41] (see Proposition 5.9).

It is the aim of this paper to continue the study of the relationship between fuzzy metric spaces and the different notions of fuzzy uniformities. The paper is organized as follows.

In Section 2 we provide all the details which are needed on fuzzy metric spaces. We presuppose some basic facts about (quasi-)uniformities. Terms and undefined concepts can be found in $[4,5]$.

Section 3 is devoted to recall the notions of probabilistic and Lowen uniformity as well as various functors between the categories Unif of uniform spaces and PUnif $(*)$ of probabilistic uniformities (and LUnif $(*)$ of Lowen uniformities).

In Section 4 we study different procedures to associate a probabilistic uniformity to a given fuzzy metric space and how they compose with the different functors introduced in the previous section. In fact, we provide four different probabilistic uniformities obtained from a fuzzy metric space $(X, M, *):\left(\mathcal{U}_{M}, *\right)$ inspired by the Hutton [0,1]-quasi-uniformity defined in [12] (see Proposition 4.1); $\mathcal{U}_{M}^{01}$ which has as a base the characteristic functions of the entourages of $\mathcal{U}_{M} ;\left(\mathcal{U}_{M}^{H}, *\right)$ motivated by the Hutton [0,1]-quasi-uniformity defined in [41]; and $\omega\left(\mathcal{U}_{M}\right)$ which is the image under Lowen's functor $\omega$ (see Theorem 3.8) of the uniformity $\mathcal{U}_{M}$ associated to the fuzzy metric space.

In Section 5, using Höhle's construction (see Proposition 5.4 and [43, Proposition $4.2]$ ), we construct four different Hutton [0,1](-quasi)-uniformities, two of them coinciding with those constructed in [12] and [41]. In this context, we show how the functors associated with these constructions behave with respect Katsaras's functors [22]. As a byproduct of our constructions we obtain a factorization of Katsaras's functor $\Phi$, which allows to obtain a Hutton [0,1](-quasi)-uniformity from a uniformity, via the category of probabilistic uniformities (see Proposition 5.16).

In Section 6, we study the topologies and stratified $I$-topologies associated with the fuzzy uniformities that we have considered. 
Finally, the last section is devoted to the study of the relationship between fuzzy metrics and fuzzifying topologies and uniformities. In particular, we develop another method of constructing a probabilistic uniformity in a fuzzy metric space by using the fuzzifying uniformity constructed in this context as in $[30,40]$. We also clarify its relationship with the other probabilistic uniformities induced by a fuzzy metric.

\section{FUZZY METRIC SPACES}

Definition 2.1 ([36]). A binary operation $*:[0,1] \times[0,1] \rightarrow[0,1]$ is called a continuous t-norm if $([0,1], *)$ is an Abelian topological monoid with unit 1 , such that $\alpha * \beta \leq \gamma * \delta$ whenever $\alpha \leq \gamma$ and $\beta \leq \delta$, with $\alpha, \beta, \gamma, \delta \in[0,1]$.

We say that the continuous $t$-norm $*$ does not have nontrivial zero divisors, if $\alpha * \beta \neq 0$ whenever $\alpha, \beta \neq 0$.

If $*$ is a continuous $t$-norm, since the map $\alpha *(\cdot):[0,1] \rightarrow[0,1]$ preserves arbitrary joins for each $\alpha \in[0,1]$, it has a right adjoint $\alpha \stackrel{*}{\rightarrow}(\cdot):[0,1] \rightarrow[0,1]$ determined by the adjoint property

$$
\alpha * \beta \leq \gamma \Longleftrightarrow \beta \leq \alpha \stackrel{*}{\rightarrow} \gamma, \quad \alpha, \beta, \gamma \in[0,1] .
$$

Hence the implication $\stackrel{*}{\rightarrow}$ is the binary operation on $[0,1]$ given by

$$
\alpha \stackrel{*}{\rightarrow} \gamma=\bigvee\{\beta \in[0,1] \mid \alpha * \beta \leq \gamma\}, \quad \alpha, c \in[0,1]
$$

(We will omit the superscript $*$ if no confusion arises).

Three distinguished examples of continuous $t$-norms are $\wedge, \cdot$ and $*_{\mathrm{E}}$ (the Eukasiewicz $t$-norm) which are defined as

$$
\alpha \wedge \beta=\min \{\alpha, \beta\}, \quad \alpha \cdot \beta=\alpha \beta \quad \text { and } \quad \alpha *_{\varepsilon} \beta=\max \{\alpha+\beta-1,0\}
$$

and the corresponding implications are defined as

$\alpha \stackrel{\rightarrow}{\rightarrow}=\left\{\begin{array}{ll}1, & \text { if } \alpha \leq \beta ; \\ \beta, & \text { if } \beta<\alpha ;\end{array} \quad \alpha \rightarrow \beta=\left\{\begin{array}{ll}1, & \text { if } \alpha \leq \beta ; \\ \frac{\beta}{\alpha}, & \text { if } \beta<\alpha ;\end{array}\right.\right.$ and $\alpha \stackrel{*_{\underline{E}}}{\rightarrow} \beta=\min \{1-\alpha+\beta, 1\}$,

for all $\alpha, \beta \in[0,1]$. It is well-known and easy to see that $* \leq \wedge$ for each continuous $t$-norm $*$.

Definition $2.2([6])$. A fuzzy metric (in the sense of George and Veeramani) on a set $X$ is a pair $(M, *)$ such that $*$ is a continuous $t$-norm and $M$ is a fuzzy set on $X \times X \times(0,+\infty)$ such that for all $x, y, z \in X, t, s>0$ :

(GV1) $M(x, y, t)>0$;

(GV2) $M(x, y, t)=1$ if and only if $x=y$;

(GV3) $M(x, y, t)=M(y, x, t)$;

(GV4) $M(x, y, t+s) \geq M(x, z, t) * M(z, y, s)$;

$(\mathrm{GV} 5) M(x, y,-):(0,+\infty) \rightarrow[0,1]$ is continuous.

In this case, $(X, M, *)$ is said to be a fuzzy metric space.

A simple but useful fact is that $M\left(x, y,{ }_{-}\right)$is nondecreasing for all $x, y \in X([8])$.

Remark 2.3. We recall that, in modern terminology, a fuzzy metric in the sense of Kramosil and Michalek (cf. [24]) is a pair $(M, *)$ such that $*$ is a continuous $t$-norm and $M$ is a fuzzy set on $X \times X \times[0,+\infty)$ satisfying (GV3), (GV4) and

(KM1) $M(x, y, 0)=0$ for all $x, y \in X$; 
(KM2) $M(x, y, t)=1$ for all $t>0$ if and only if $x=y$;

$(\mathrm{KM} 5) \quad M(x, y,-):[0, \infty) \rightarrow[0,1]$ is left continuous.

Observe that if $(M, *)$ is a fuzzy metric then we can construct a fuzzy metric in the sense of Kramosil and Michalek $\left(M^{\prime}, *\right)$ defining $M^{\prime}(x, y, 0)=0$ and $M^{\prime}(x, y, t)=$ $M(x, y, t)$ for all $x, y \in X$ and all $t>0$.

Example 2.4. Let $(X, d)$ be a metric space and $*$ be a continuous $t$-norm. Let $M_{d}$ be the function defined on $X \times X \times(0, \infty)$ by

$$
M_{d}(x, y, t)=\frac{t}{t+d(x, y)}
$$

for all $x, y \in X$ and $t>0$.

Then $\left(X, M_{d}, *\right)$ is a fuzzy metric space called the standard fuzzy metric space (see $[6])$, and $\left(M_{d}, *\right)$ will be called the standard fuzzy metric of $d$.

George and Veeramani proved in [6] that every fuzzy metric $(M, *)$ on $X$ generates a topology $\tau(M)$ on $X$ which has as a base the family $\left\{B_{M}(x, \varepsilon, t) \mid x \in\right.$ $X, 0<\varepsilon<1, t>0\}$, where $B_{M}(x, \varepsilon, t)=\{y \in X \mid M(x, y, t)>1-\varepsilon\}$ for all $\varepsilon \in(0,1)$ and $t>0$. They also proved that $(X, \tau(M))$ is a Hausdorff first countable topological space. Moreover, if $(X, d)$ is a metric space, then the topology generated by $d$ coincides with the topology $\tau\left(M_{d}\right)$ generated by the fuzzy metric $\left(M_{d}, *\right)$.

Remark 2.5. In [9] it was proved that every fuzzy metric space $(X, M, *)$ is metrizable and possesses a compatible uniformity $\mathcal{U}_{M}$ with a countable base given by

$$
U_{n}^{M}=\left\{(x, y) \in X \times X \mid M\left(x, y, \frac{1}{n}\right)>1-\frac{1}{n}\right\}
$$

(we will omit the superscript $M$ if no confusion arises).

Furthermore, we notice that if $(X, d)$ is a metric space then $\mathcal{U}_{d}=\mathcal{U}_{M_{d}}[10$, Lemma $5]$ where $\mathcal{U}_{d}$ is the uniformity generated by $d$ which has as a base the entourages of the form $U_{d, \varepsilon}=\{(x, y) \in X \times X \mid d(x, y)<\varepsilon\}$ for every $\varepsilon>0$.

Remark 2.6. Observe that if $\left(M^{\prime}, *\right)$ is a fuzzy metric in the sense of Kramosil and Michalek then we can construct as above a topology $\tau_{M^{\prime}}$ and a uniformity $\mathcal{U}_{M^{\prime}}$ [11]. Then if $(M, *)$ is a fuzzy metric on $X$ and $\left(M^{\prime}, *\right)$ is its associated fuzzy metric in the sense of Kramosil and Michalek we can easily see that $\mathcal{U}_{M}=\mathcal{U}_{M^{\prime}}$.

Furthermore, if $d$ is a pseudometric compatible with the uniformity $\mathcal{U}_{M^{\prime}}$ then $\mathcal{U}_{M_{d}}=\mathcal{U}_{M^{\prime}}$

Consequently, fuzzy metrics in the sense of George and Veeramani and in the sense of Kramosil and Michalek are uniformly equivalent in this sense. Hence, the results that we obtain in Section 4 about probabilistic uniformities generated by means of the uniformity of a fuzzy metric remain valid if we consider Kramosil and Michalek fuzzy metrics. Moreover, Proposition 4.1 is also fulfilled in this context. Therefore, all results of the paper are true regardless of the type of fuzzy metric considered.

Definition $2.7([7])$. Let $(X, M, *)$ and $(Y, N, \star)$ be two fuzzy metric spaces. A mapping $f: X \rightarrow Y$ is said to be uniformly continuous if for every $\varepsilon \in(0,1)$ and $t>0$ there exist $\delta \in(0,1)$ and $s>0$ such that

$$
\text { if } M(x, y, s)>1-\delta \text { then } N(f(x), f(y), t)>1-\varepsilon
$$

for all $x, y \in X$. 
It is straightforward to check that $f:(X, M, *) \rightarrow(Y, N, \star)$ is uniformly continuous if and only if $f:\left(X, \mathcal{U}_{M}\right) \rightarrow\left(Y, \mathcal{U}_{N}\right)$ is uniformly continuous [10, Lemma 1].

With all these facts, we can consider the categories FMetric, FMetric $(*)$, Metric, and Unif whose objects are, respectively, the fuzzy metric spaces, the fuzzy metric spaces with a given continuous $t$-norm $*$, the metric spaces and the uniform spaces, and whose morphisms, in all of them, are the uniformly continuous functions. Then the functor $\Pi_{*}$ : Metric $\rightarrow$ FMetric $(*)$ (resp. $\Upsilon$ : FMetric $\rightarrow$ Unif) which leaves morphisms unchanged and $\Pi_{*}((X, d))=\left(X, M_{d}, *\right)\left(\right.$ resp. $\left.\Upsilon((X, M, *))=\left(X, \mathcal{U}_{M}\right)\right)$ is fully faithful.

\section{Probabilistic Uniformities}

Although some of the definitions that we will use were originally defined in a wider lattice-theoretical context, we will restrict ourselves to the closed unit interval $I=[0,1]$ since the fuzzy metric spaces that we will consider depend on fuzzy sets with values in $I$. We will also use the following notation: $I_{0}=(0,1]$ and $I_{1}=[0,1)$. Given a nonempty set $X$, let us denote by $1_{A}$ the characteristic function of a subset $A$ of $X$.

Definition $3.1([26])$. Let $X$ be a nonempty set. A family $\mathcal{F}$ of subsets of $I^{X}$ is called a prefilter on $X$ if it is a filter of the lattice $I^{X}$, that is:

(P1) given $a, b \in I^{X}$ such that $a \leq b$ and $a \in \mathcal{F}$ then $b \in \mathcal{F}$;

$(\mathrm{P} 2)$ if $a, b \in \mathcal{F}$ then $a \wedge b \in \mathcal{F}$;

(P3) $1_{\varnothing} \notin \mathcal{F}$.

A subfamily $\mathcal{B}$ of $\mathcal{F}$ is a prefilter base for the prefilter $\mathcal{F}$ if for every $a \in \mathcal{F}$ there exists $b \in \mathcal{B}$ such that $b \leq F$.

It is clear that every family $\mathcal{B}$ of subsets of $I^{X}$ satisfying

$$
\text { for every } a, b \in \mathcal{B} \text { there exists } c \in \mathcal{B} \text { with } c \leq a \wedge b
$$

is a prefilter base for the prefilter $\mathcal{F}$ of all fuzzy sets $a$ on $X$ for which there exists $b \in \mathcal{B}$ with $b \leq a$.

Following the terminology of [16], we recall the following definition.

Definition 3.2 ([16, Definition 2.1], [21]). A probabilistic uniformity on a nonempty set $X$ is a pair $(\mathcal{U}, *)$, where $*$ is a continuous $t$-norm and $\mathcal{U}$ is a prefilter on $X \times X$ such that:

(PU1) $U(x, x)=1$ for all $U \in \mathcal{U}$ and $x \in X$;

(PU2) if $U \in \mathcal{U}$ then $U^{-1} \in \mathcal{U}$ where $U^{-1}(x, y)=U(y, x)$;

(PU3) for each $U \in \mathcal{U}$ there exists $V \in \mathcal{U}$ such that

$$
V^{2} \leq U
$$

where $V^{2}(x, y)=\bigvee_{z \in X} V(x, z) * V(z, y)$;

In this case, the pair $(X, \mathcal{U}, *)$ is called a probabilistic uniform space.

A function $f:(X, \mathcal{U}, *) \rightarrow(Y, \mathcal{V}, \star)$ between two probabilistic uniform spaces is said to be uniformly continuous if $(f \times f)^{-1}(V) \in \mathcal{U}$ for all $V \in \mathcal{V}$, i.e. for every $V \in \mathcal{V}$ there exists $U \in \mathcal{U}$ such that

$$
U(x, y) \leq V(f(x), f(y)) \text { for all } x, y \in X .
$$

We denote by PUnif the category of probabilistic uniform spaces and uniformly continuous functions. For a fixed continuous $t$-norm, $\operatorname{PUnif}(*)$ is the full subcategory of PUnif whose objects are the probabilistic uniformities with respect to $*$. 
Note that probabilistic uniformities are called Höhle-Katsaras uniformities in [42].

Definition 3.3. If $(\mathcal{U}, *)$ is a probabilistic uniformity on $X$, the pair $(\mathcal{B}, *)$ is said to be a base for $(\mathcal{U}, *)$ if $\mathcal{B}$ is a prefilter base for the prefilter $\mathcal{U}$.

Each prefilter base $\mathcal{B}$ on $X \times X$ satisfying (PU1), (PU3) and (BU2) given $B \in \mathcal{B}$ there exists $B^{\prime} \in \mathcal{B}$ with $B^{\prime}(x, y) \leq B(y, x)$ for all $x, y \in X$, generates a probabilistic uniformity $(\mathcal{U}, *)$ on $X$ where $\mathcal{U}$ is the filter generated by B.

Proposition 3.4. Let $(X, \mathcal{U})$ be a uniform space and $(X, \mathcal{U}, *)$ be a probabilistic uniform space. Let us denote:

(1) $\Gamma(\mathcal{U})=\mathcal{U}_{01}$ is the prefilter on $X \times X$ generated by $\left\{1_{U} \mid U \in \mathcal{U}\right\}$;

(2) $\Theta(\mathcal{U})=\mathcal{U}_{01}$ is the filter $\left\{U \subseteq X \times X \mid 1_{U} \in \mathcal{U}\right\}$.

Then:

(i) $\Gamma_{*}$ : Unif $\rightarrow$ PUnif $(*)$ is a fully faithful functor sending each $(X, \mathcal{U})$ to $\left(X, \mathcal{U}_{01}, *\right)$ and leaving morphisms unchanged;

(ii) If $*$ does not have nontrivial zero divisors, then $\Theta_{*}$ : PUnif $(*) \rightarrow$ Unif is a faithful functor sending each $(X, \mathcal{U}, *)$ to $\left(X, \mathcal{U}_{01}\right)$ and leaving morphisms unchanged.

Furthermore, $\Theta_{*} \circ \Gamma_{*}=1_{\text {Unif }}$.

Proof. (i) It is easy to prove that $\left\{1_{U} \mid U \in \mathcal{U}\right\}$ is a prefilter base for a probabilistic uniformity $\left(\mathcal{U}_{01}, *\right)$. Furthermore, if $(X, \mathcal{U})$ and $(Y, \mathcal{V})$ are two uniform spaces then $f:(X, \mathcal{U}) \rightarrow(Y, \mathcal{V})$ is uniformly continuous if and only if $f:\left(X, \mathcal{U}_{01}, *\right) \rightarrow\left(Y, \mathcal{V}_{01}, *\right)$ is uniformly continuous. Consequently, $\Gamma_{*}$ is fully faithful.

(ii) It is clear that $\mathcal{U}_{01}$ is a filter. Furthermore, if $1_{U} \in \mathcal{U}$ then $\Delta \subseteq U$ by (PU1) and $U \in \mathcal{U}_{01}$ implies $U^{-1} \in \mathcal{U}_{01}$ by (PU2). Finally, suppose that $1_{U} \in \mathcal{U}$. Then there exists $F \in \mathcal{U}$ such that $F^{2} \leq 1_{U}$. Let $V=\{(x, y) \in X \times X \mid F(x, y) \neq 0\}$. It is clear that $F \leq 1_{V}$ so $1_{V} \in \mathcal{U}$. We show that $V^{2} \subseteq U$. Suppose that $(x, z),(z, y) \in V$. Hence $F(x, z) \neq 0$ and $F(z, y) \neq 0$ so $F(x, z) * F(z, y) \neq 0$. Since $F^{2} \leq 1_{U}$ and $F^{2}(x, y)>0$ then $1_{U}(x, y)=1$, i.e. $(x, y) \in U$. Hence $\mathcal{U}_{01}$ is a uniformity.

Now suppose that $f:(X, \mathcal{U}, *) \rightarrow(Y, \mathcal{V}, *)$ is a uniformly continuous function between two probabilistic uniform spaces. Let $V \in \mathcal{V}_{01}$. Then $1_{V} \in \mathcal{V}$ so we can find $U \in \mathcal{U}$ with $U(x, y) \leq 1_{V}(f(x), f(y))$ for all $(x, y) \in X \times X$. It is easy to see that $1_{(f \times f)^{-1}(V)} \in \mathcal{U}$ and so $(f \times f)^{-1}(V) \in \mathcal{U}_{01}$. Hence $f:\left(X, \mathcal{U}_{01}\right) \rightarrow\left(Y, \mathcal{V}_{01}\right)$ is uniformly continuous which implies that $\Theta_{*}$ is faithful.

Finally, it is obvious that $\Theta_{*} \circ \Gamma_{*}=1_{\text {Unif }}$.

Remark 3.5. Observe that if $\mathcal{B}$ is a base for a uniformity $\mathcal{U}$ on $X$ then $\left\{1_{B} \mid B \in \mathcal{B}\right\}$ is a prefilter base for $\mathcal{U}_{01}$.

In 1981, Lowen introduced, for the $t$-norm $\wedge$, the following notion of fuzzy uniformity which is very related with that of probabilistic uniformity.

Definition 3.6 ([27], cf. [18, Definition 4.1]). A Lowen uniformity on a nonempty set $X$ is a pair $(\mathcal{U}, *)$, where $*$ is a continuous $t$-norm and $\mathcal{U}$ is a prefilter on $X \times X$ such that:

(LU1) $U(x, x)=1$ for all $U \in \mathcal{U}$ and $x \in X$;

(LU2) if $U \in \mathcal{U}$ then $U^{-1} \in \mathcal{U}$ where $U^{-1}(x, y)=U(y, x)$; 
(LU3) for each $U \in \mathcal{U}$ and each $\varepsilon \in I_{0}$ there exists $V \in \mathcal{U}$ such that

$$
V^{2}-\varepsilon \leq U
$$

where $V^{2}(x, y)=\bigvee_{z \in X} V(x, z) * V(z, y)$;

(LU4) $\bigvee_{\varepsilon \in I_{0}}\left(U_{\varepsilon}-\varepsilon\right) \in \mathcal{U}$ for each family $\left\{U_{\varepsilon} \mid \varepsilon \in I_{0}\right\} \subseteq \mathcal{U}$, i.e. $\mathcal{U}$ is saturated.

In this case, the pair $(X, \mathcal{U}, *)$ is called a Lowen uniform space.

As in the case of probabilistic uniform spaces, we can consider the category LUnif whose objects are the Lowen uniform spaces and whose morphism are the uniformly continuous functions as defined in the obvious way (see [27, Definition 2.4]).

Remark 3.7. We observe that, in general, if $(X, \mathcal{U})$ is a uniform space then $\Gamma_{*}(X, \mathcal{U})=$ $\left(X, \mathcal{U}_{01}, *\right)$ is not a Lowen uniform space. If fact, let us consider on $\mathbb{R}$ the uniformity $\mathcal{U}_{e}$ generated by the subsets $U_{\varepsilon}=\left\{(x, y) \in \mathbb{R}^{2}|| x-y \mid<\varepsilon\right\}$ for all $\varepsilon>0$. For each $\delta \in I_{0}$ we have that $1_{U_{\delta}}\left(x, x+\frac{\delta}{2}\right)=1$ but $\bigvee_{\varepsilon \in I_{0}}\left(1_{U_{\varepsilon}}-\varepsilon\right)\left(x, x+\frac{\delta}{2}\right)=1-\frac{\delta}{2}$ and so $1_{U_{\delta}} \not \bigvee_{\varepsilon \in I_{0}}\left(1_{U_{\varepsilon}}-\varepsilon\right)$ for all $\delta>0$. Hence $\bigvee_{\varepsilon \in I_{0}}\left(1_{U_{\varepsilon}}-\varepsilon\right) \notin\left(\mathcal{U}_{e}\right)_{01}$.

In [27], Lowen defined two functors to establish a relation between classical uniformities and Lowen uniformities as follows:

Theorem $3.8([27])$. Let $X$ be a nonempty set, $\mathcal{U}$ be a uniformity on $X$ and $(\mathcal{U}, *)$ be a Lowen uniformity on $X$. Define

$$
\begin{aligned}
\omega(\mathcal{U}) & =\left\{U \in I^{X \times X} \mid U^{-1}((\alpha, 1]) \in \mathcal{U} \text { for all } \alpha \in I_{1}\right\} \quad \text { and } \\
\iota(\mathcal{U}) & =\left\{U^{-1}((\alpha, 1]) \mid U \in \mathcal{U}, \alpha \in I_{1}\right\} .
\end{aligned}
$$

Then:

(1) $(\omega(\mathcal{U}), *)$ is a Lowen uniformity on $X$;

(2) $\iota(\mathcal{U})$ is a uniformity on $X$;

(3) $\iota(\omega(\mathcal{U}))=\mathcal{U}$;

(4) $(\omega(\iota(\mathcal{U})), *)$ is the coarsest Lowen uniformity generated by a uniformity and which is finer than $\mathcal{U}$.

Furthermore, the functor $\omega_{*}:$ Unif $\rightarrow \operatorname{LUnif}(*)$ given by $\omega_{*}((X, \mathcal{U}))=(X, \omega(\mathcal{U}), *)$ and which leaves morphisms unchanged is fully faithful while the functor $\iota:$ LUnif $\rightarrow$ Unif given by $\iota((X, \mathcal{U}, *))=(X, \iota(\mathcal{U}))$ and which leaves morphisms unchanged is faithful. Then Unif is isomorphic to a full subcategory of $\operatorname{LUnif}(*)$.

Remark 3.9. The relationship between the categories LUnif and PUnif was established in [42] where it is shown that Lowen uniformities are those probabilistic uniformities which satisfy (LU4), i.e. the Lowen uniformities are the saturated probabilistic uniformities. In this way, LUnif is a concretely coreflective full subcategory of PUnif. In fact, if $(X, \mathcal{U}, *)$ is a probabilistic uniform space then its correflection is the Lowen uniform space $(X, \widetilde{\mathcal{U}}, *)$, where $\widetilde{\mathcal{U}}$ is the saturation of $\mathcal{U}$ given by

$$
\widetilde{\mathcal{U}}=\left\{\bigvee_{\varepsilon \in I_{0}}\left(U_{\varepsilon}-\varepsilon\right) \mid U_{\varepsilon} \in \mathcal{U} \text { for all } \varepsilon \in I_{0}\right\} .
$$

Observe that $(\widetilde{\mathcal{U}}, *)$ is the coarsest Lowen uniformity finer than $(\mathcal{U}, *)$. On the other hand, if $f:(X, \mathcal{U}, *) \rightarrow(Y, \mathcal{V}, \star)$ is a uniformly continuous function between two probabilistic uniform spaces then so is $f:(X, \widetilde{\mathcal{U}}, *) \rightarrow(Y, \widetilde{\mathcal{V}}, \star)$. Consequently we have a functor $\mathcal{S}: \operatorname{PUnif}(*) \rightarrow \operatorname{LUnif}(*)$ given by $\mathcal{S}((X, \mathcal{U}, *))=(X, \widetilde{\mathcal{U}}, *)$ and which leaves morphisms unchanged 
The following result factorizes Lowen's functor $\omega_{*}$ by means of the functors $\Gamma_{*}$ and $\mathcal{S}$.

Proposition 3.10. The following diagram commutes

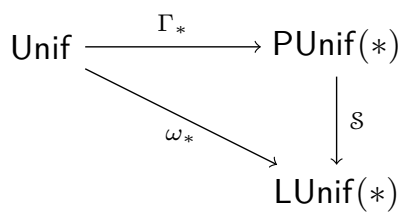

Proof. We only have to prove that $\omega(\mathcal{U})=\widetilde{\mathcal{U}_{01}}$ for each uniform space $(X, \mathcal{U})$. Let $U \in \omega(\mathcal{U})$. Then $U^{-1}((\alpha, 1]) \in \mathcal{U}$ for all $\alpha \in(0,1)$ and it follows that $\bigvee_{\varepsilon \in I_{0}}\left(1_{U^{-1}((1-\varepsilon, 1])}-\varepsilon\right)=U \in \widetilde{\mathcal{U}_{01}}$.

On the other hand, if $U_{\varepsilon} \in \mathcal{U}$ for each $\varepsilon \in I_{0}$ and $\widetilde{U}=\bigvee_{\varepsilon \in I_{0}}\left(1_{U_{\varepsilon}}-\varepsilon\right)$, then $\widetilde{U}^{-1}((\alpha, 1])=\bigcup\left\{U_{\varepsilon} \mid 1-\varepsilon>\alpha\right\} \in \mathcal{U}$ for each $\alpha \in I_{1}$ and so $\widetilde{U} \in \omega(\mathcal{U})$.

The following result establishes the relationship between the pairs of functors $\omega_{*}, \Theta_{*}$ and $\iota, \Gamma_{*}$.

Proposition 3.11. Let $X$ be a nonempty set, $(\mathcal{U}, *)$ be a Lowen uniformity on $X$ and $\mathcal{U}$ be a uniformity on $X$. If $*$ does not have nontrivial zero divisors, then:

(1) $\Theta(\mathcal{U}) \subseteq \iota(\mathcal{U})$.

(2) $\omega(\Theta(\mathcal{U})) \subseteq \widetilde{U} \subseteq \omega(\iota(\mathcal{U}))$.

(3) $\Theta(\omega(\mathcal{U}))=\mathcal{U}=\iota(\Gamma(\mathcal{U}))$.

Proof. (1) This is obvious since given $U \subseteq X \times X$ with $1_{U} \in \mathcal{U}$ then $U=$ $1_{U}^{-1}((0,1]) \in \iota(\mathcal{U})$.

(2) Let $U \in \omega(\Theta(\mathcal{U}))$. Then $U^{-1}((\alpha, 1]) \in \Theta(\mathcal{U})$ for all $\alpha \in I_{1}$, i.e. $1_{U^{-1}((\alpha, 1])} \in \mathcal{U}$ for all $\alpha \in I_{1}$. Since $U=\bigvee_{\varepsilon \in I_{0}}\left(1_{U^{-1}((1-\varepsilon, 1])}-\varepsilon\right)$ and $\tilde{\mathcal{U}}$ is a Lowen uniformity then $U \in \tilde{U}$.

The second inclusion is obvious since $\widetilde{U}$ is the coarsest Lowen uniformity finer than $\mathcal{U}$ and $\omega(\iota(\mathcal{U}))$ is the coarsest Lowen uniformity finer than $\mathcal{U}$ generated by a uniformity (see Theorem 3.8(4)).

(3) This follows from the following easy equivalences for each $U \subseteq X \times X$ :

$$
\begin{aligned}
U \in \mathcal{U} & \Longleftrightarrow 1_{U} \in \omega(\mathcal{U}) \Longleftrightarrow U \in \Theta(\omega(\mathcal{U})) \\
& \Longleftrightarrow 1_{U} \in \Gamma(\mathcal{U}) \Longleftrightarrow U \in \iota(\Gamma(\mathcal{U})) .
\end{aligned}
$$

\section{Probabilistic Uniformities INDUCED BY A FUZZY METRIC SPACE}

In the following we will introduce four different probabilistic uniformities in a fuzzy metric space.

We observe that if $(X, M, *)$ is a fuzzy pseudometric space then the family $\left\{M_{t}\right.$ $t>0\}$ is a base for a probabilistic uniformity $\left(\mathcal{U}_{M}^{H}, *\right)$ on $X[16$, Theorem 3.3] and for a Lowen uniformity $\left(\widetilde{\mathcal{U}_{M}^{H}}, *\right)[19$, Theorem 2.6]. This way of generating fuzzy uniformities from a fuzzy metric space is important since it allows to establish some categorical isomorphism theorems [33].

The construction given in the following result is motivated by the Hutton $[0,1]$ (quasi-)uniformity constructed in [12]. 
Proposition 4.1. Let $(X, M, *)$ be a fuzzy metric space. For each $t>0$ and each $\varepsilon \in I_{0}$ we denote by $U_{\varepsilon, t}^{M}$ the fuzzy set on $X \times X$ given by:

$U_{\varepsilon, t}^{M}(x, y)=(1-\varepsilon) \rightarrow M(x, y, t)=\bigvee\{\lambda \in I \mid(1-\varepsilon) * \lambda \leq M(x, y, t)\}, \quad x, y \in X$, and $\mathcal{B}_{M}=\left\{U_{\varepsilon, t}^{M} \mid \varepsilon \in I_{0}, t>0\right\}$. Then $\mathcal{B}_{M}$ is a prefilter base generating a probabilistic uniformity $\mathfrak{U}_{M}$ on $X$.

Proof. It is easy to see that $\mathcal{B}_{M}$ is a prefilter base. Indeed, given $\varepsilon, \delta \in I_{0}$ and $s, t>0$ then $U_{\gamma, r}^{M} \leq U_{\varepsilon, t}^{M} \wedge U_{\delta, s}^{M}$ where $\gamma=\min \{\varepsilon, \delta\} \in I_{0}$ and $r=\min \{s, t\}>0$. Let $U_{\varepsilon, t}^{M} \in \mathcal{B}_{M}$. Then $U_{\varepsilon, t}^{M}(x, x)=(1-\varepsilon) \rightarrow M(x, x, t)=(1-\varepsilon) \rightarrow 1=1$ so (PU1) is verified. On the other hand, $\left(U_{\varepsilon, t}^{M}\right)^{-1}=U_{\varepsilon, t}^{M}$ for all $\varepsilon \in I_{0}$ and $t>0$, so (PU2), and in particular (BU2), is fulfilled. Finally, given $\varepsilon \in I_{0}$ let $\delta \in I_{0}$ such that $1-\varepsilon \leq(1-\delta) *(1-\delta)$. Then

$$
\left(U_{\delta, \frac{t}{2}}^{M}\right)^{2} \leq U_{\varepsilon, t}^{M}
$$

In fact, let $x, y, z \in X$. Since $(1-\delta) * U_{\delta, \frac{t}{2}}^{M}(x, z) \leq M\left(x, z, \frac{t}{2}\right)$ and $(1-\delta) * U_{\delta, \frac{t}{2}}^{M}(z, y) \leq$ $M\left(z, y, \frac{t}{2}\right)$ it follows that

$$
\begin{aligned}
(1-\varepsilon) * U_{\delta, \frac{t}{2}}^{M}(x, z) * U_{\delta, \frac{t}{2}}^{M}(z, y) & \leq(1-\delta) *(1-\delta) * U_{\delta, \frac{t}{2}}^{M}(x, z) * U_{\delta, \frac{t}{2}}^{M}(z, y) \\
& \leq M\left(x, z, \frac{t}{2}\right) * M\left(z, y, \frac{t}{2}\right) \leq M(x, y, t) .
\end{aligned}
$$

Therefore

$$
\left(U_{\delta, \frac{t}{2}}^{M}\right)^{2}(x, y)=\bigvee_{z \in X} U_{\delta, \frac{t}{2}}^{M}(x, z) * U_{\delta, \frac{t}{2}}^{M}(z, y) \leq(1-\varepsilon) \rightarrow M(x, y, t)=U_{\varepsilon, t}^{M}(x, y) .
$$

Remark 4.2. We observe that the mappings $\Lambda^{H}, \Lambda$ : FMet $\rightarrow$ PUnif acting on objects as follows:

$$
\Lambda^{H}((X, M, *))=\left(X, \mathcal{U}_{M}^{H}, *\right) \quad \text { and } \quad \Lambda((X, M, *))=\left(X, \mathcal{U}_{M}, *\right)
$$

are not functors when we consider that morphisms are unchanged. Let us see an example.

Let $e$ be the euclidean metric on $\mathbb{R}$ and $*_{\mathrm{E}}$ be the Łukasiewicz $t$-norm. We observe that then $\alpha \rightarrow \beta=\min \{\beta-\alpha+1,1\}$ for all $\alpha, \beta \in I$. Let us consider the fuzzy metric spaces $\left(\mathbb{R}, N, *_{\mathrm{E}}\right)$ and $\left(\mathbb{R}, M_{e}, *_{\mathrm{E}}\right)$, where $N$ is the fuzzy set on $\mathbb{R} \times \mathbb{R} \times(0, \infty)$ given by $N(x, y, t)=\max \left\{M_{e}(x, y, t), \frac{1}{2}\right\}$. Next we show that the identity function id: $\left(\mathbb{R}, N, *_{\mathrm{E}}\right) \rightarrow\left(\mathbb{R}, M_{e}, *_{\mathrm{E}}\right)$ is uniformly continuous. In fact, let $\varepsilon \in(0,1)$ and $t>0$. Let $\delta>0$ with $1-\delta>\max \left\{\frac{1}{2}, 1-\varepsilon\right\}$. Then if $N(x, y, t)>1-\delta$ we have that $M_{e}(x, y, t)>1-\delta \geq 1-\varepsilon$ so the conclusion follows.

Nevertheless, id: $\left(\mathbb{R}, \mathfrak{U}_{N}, *_{€}\right) \rightarrow\left(\mathbb{R}, \mathfrak{U}_{M_{e}, *_{乇}}\right)$ is not uniformly continuous. Indeed, let $\varepsilon \in\left(0, \frac{1}{2}\right)$ and $t>0$. It is obvious that there exist $x, y \in \mathbb{R}$ such that $M_{e}(x, y, t)+\varepsilon<\frac{1}{2}$ so $M_{e}(x, y, t)<1-\varepsilon$ which implies $U_{\varepsilon, t}^{M_{e}}(x, y)=M_{e}(x, y, t)+\varepsilon$. Then for each $\delta \in I_{0}$ and $s>0$ we have that

$$
U_{\varepsilon, t}^{M_{e}}(x, y)=M_{e}(x, y, t)+\varepsilon<\frac{1}{2} \leq N(x, y, s) \leq(1-\delta) \rightarrow N(x, y, s)=U_{\delta, s}^{N}(x, y) .
$$

Hence $U_{\delta, s}^{N}(x, y) \not \leq U_{\varepsilon, t}^{M_{e}}(x, y)=U_{\varepsilon, t}^{M_{e}}(\operatorname{id}(x), \operatorname{id}(y))$ for every $\delta \in I_{0}$ and $s>0$ so id: $\left(\mathbb{R}, \mathcal{U}_{N}, *_{\mathrm{E}}\right) \rightarrow\left(\mathbb{R}, \mathcal{U}_{M_{e}}, *_{\mathrm{E}}\right)$ is not uniformly continuous.

Furthermore, since $M_{e}(x, y, t)<\frac{1}{2} \leq N(x, y, s)$ for all $s>0$ then we also deduce that id: $\left(\mathbb{R}, \mathcal{U}_{N}^{H}, *_{\mathrm{E}}\right) \rightarrow\left(\mathbb{R}, \mathcal{U}_{M_{e}}^{H}, *_{\mathrm{E}}\right)$ is not uniformly continuous. 
On the other hand, if $(X, M, *)$ and $(Y, N, \star)$ are two fuzzy metric spaces and $f:\left(X, \mathcal{U}_{M}, *\right) \rightarrow\left(Y, \mathcal{U}_{N}, \star\right)$ is uniformly continuous or $f:\left(X, \mathcal{U}_{M}^{H}, *\right) \rightarrow\left(Y, \mathcal{U}_{N}^{H}, \star\right)$ is uniformly continuous then $f:(X, M, *) \rightarrow(Y, N, \star)$ is uniformly continuous. We only prove the first assertion since the other follows similarly. Let $t>0$ and $\varepsilon \in I_{0}$. By assumption we can find $\delta \in I_{0}$ and $s>0$ such that

$$
U_{\delta, s}^{M}(x, y) \leq U_{\frac{\varepsilon}{2}, t}^{N}(f(x), f(y))
$$

for all $x, y \in X$. Consequently, if $M(x, y, s)>1-\delta$ then $U_{\frac{\varepsilon}{2}, t}^{N}(f(x), f(y)) \geq$ $U_{\delta, s}^{M}(x, y)=1$ so $N(f(x), f(y), t) \geq 1-\frac{\varepsilon}{2}>1-\varepsilon$. Hence $f:(X, M, *) \rightarrow(Y, N, \star)$ is uniformly continuous.

Example 4.3. Let $(X, d)$ be a metric space. Then the probabilistic uniformity $\left(\mathcal{U}_{M_{d}}, \cdot\right)$ induced by the standard fuzzy metric $\left(M_{d}, \cdot\right)$ has as a base the fuzzy sets $U_{\varepsilon, t}^{d}$ given by

$$
U_{\varepsilon, t}^{d}(x, y)=(1-\varepsilon) \rightarrow M_{d}(x, y, t)=(1-\varepsilon) \rightarrow \frac{t}{t+d(x, y)}=\min \left\{\frac{t}{(t+d(x, y))(1-\varepsilon)}, 1\right\}
$$

for all $\varepsilon \in(0,1), t>0$ and $x, y \in X$.

Notation 4.4. Let $(X, M, *)$ be a fuzzy metric space. Then, by Proposition 3.4, $\Gamma_{*}\left(\left(X, \mathcal{U}_{M}\right)\right)=\left(X,\left(\mathcal{U}_{M}\right)_{01}, *\right)$ is a probabilistic uniform space whose probabilistic uniformity will be denoted by $\left(\mathcal{U}_{M}^{01}, *\right)$.

Let $(M, *)$ be a fuzzy metric on $X$. Using Theorem 3.8 , we have that $\left(\omega\left(\mathcal{U}_{M}\right), *\right)$ is a Lowen uniformity associated to a fuzzy metric space (see Remark 3.9).

Remark 4.5. Let $(X, d)$ be a metric space. As we have previously observed in Remark 2.5, $\mathcal{U}_{d}=\mathcal{U}_{M_{d}}$ so

$$
\left(X,\left(\mathcal{U}_{d}\right)_{01}, *\right)=\left(X, \mathcal{U}_{M_{d}}^{01}, *\right) \quad \text { and } \quad\left(X, \omega\left(\mathcal{U}_{d}\right), *\right)=\left(X, \omega\left(\mathcal{U}_{M_{d}}\right), *\right)
$$

for every continuous $t$-norm $*$.

The following result gives the relationship between the four probabilistic uniformities we have introduced.

Proposition 4.6. Let $(X, M, *)$ be a fuzzy metric space. Then

$$
\mathcal{U}_{M} \subseteq \mathcal{U}_{M}^{01} \wedge \mathcal{U}_{M}^{H} \subseteq \mathcal{U}_{M}^{01} \vee \mathcal{U}_{M}^{H} \subseteq \omega\left(\mathcal{U}_{M}\right)
$$

Proof. Let $n \in \mathbb{N}$ and $U_{n}^{M}=\left\{(x, y) \in X \times X \mid M\left(x, y, \frac{1}{n}\right)>1-\frac{1}{n}\right\} \in \mathcal{U}_{M}$. Then it is obvious that $1_{U_{n}^{M}} \leq U_{\frac{1}{n}, \frac{1}{n}}^{M}$ since if $1_{U_{n}^{M}}(x, y)=1$ then $M\left(x, y, \frac{1}{n}\right)>1-\frac{1}{n}$ so $U_{\frac{1}{n}, \frac{1}{n}}^{M}(x, y)=1$. Consequently, $\mathcal{U}_{M} \subseteq \mathcal{U}_{M}^{01}$.

On the other hand, the inclusion $\mathcal{U}_{M} \subseteq \mathcal{U}_{M}^{H}$ follows immediately since $M(x, y, t) \leq$ $(1-\varepsilon) \rightarrow M(x, y, t)$ for all $x, y \in X, t>0$ and $\varepsilon \in I_{0}$.

For the last inclusion, let $U \in \mathcal{U}_{M}$. Then $1_{U}^{-1}((\varepsilon, 1])=U$ for all $\varepsilon \in I_{1}$. Hence $1_{U}^{-1}((\varepsilon, 1]) \in \mathcal{U}_{M}$ for all $\varepsilon \in I_{1}$ so $1_{U} \in \omega\left(\mathcal{U}_{M}\right)$ which proves $\mathcal{U}_{M}^{01} \subseteq \omega\left(\mathcal{U}_{M}\right)$.

Furthermore, $\mathcal{U}_{M}^{H} \subseteq \omega\left(\mathcal{U}_{M}\right)$ since $M_{t}^{-1}(\varepsilon, 1]=\{(x, y) \in X \times X \mid M(x, y, t)>\varepsilon\} \in$ $\mathcal{U}_{M}$ for all $t>0$ and $\varepsilon \in I_{1}$.

The following examples show that the above inclusions are the only ones, in general. 
Example 4.7. Let $(X, M, *)$ be a fuzzy metric space such that $\mathcal{U}_{M} \neq\{X \times X\}$ (it suffices to find $x, y \in X$ and $t>0$ with $M(x, y, t)<1)$. We notice that $0<U_{\varepsilon, t}^{M}(x, y)=(1-\varepsilon) \rightarrow M(x, y, t)$ for all $\varepsilon \in I_{0}, x, y \in X$ and $t>0$ since $M(x, y, t)>0$. Consequently, given $X \times X \neq U \in \mathcal{U}_{M}$ it follows that $1_{U} \notin \mathcal{U}_{M}$. In fact, suppose that $(x, y) \notin U$. Then $1_{U}(x, y)=0$ but $U_{\varepsilon, t}(x, y)>0$ for all $\varepsilon \in I_{0}$ and $t>0$. Therefore, $\mathcal{U}_{M}^{01} \nsubseteq \mathcal{U}_{M}$.

This also shows that $\mathcal{U}_{M}^{01} \nsubseteq \mathcal{U}_{M}^{H}$ since as above $1_{U}(x, y)=0$ but $M_{t}(x, y) \neq 0$ for all $t>0$.

Example 4.8. Let us consider the euclidean metric $e$ on $I$ and the fuzzy metric space $\left(I, M, *_{\mathrm{E}}\right)$ where $M(x, y, t)=1-e(x, y)$ for all $x, y \in I$ and all $t>0$. Given $\varepsilon \in I_{0}$ we can find $x, y \in I$ such that $0<e(x, y)<\varepsilon$. Hence $(1-\varepsilon) \rightarrow M(x, y, t)=$ $1>M(x, y, t)$ so $\mathcal{U}_{M}^{H} \nsubseteq \mathcal{U}_{M}$.

Example 4.9. Let $(X, M, *)$ be a fuzzy metric space such that $\mathcal{U}_{M}$ is not the discrete uniformity, i.e. there exists a superset of the diagonal of $X$ which does not belong to $\mathcal{U}_{M}$. Define $M_{t} \in I^{X \times X}$ by $M_{t}(x, y)=M(x, y, t)$ for all $t>0$. It is clear that $M_{t} \in \omega\left(\mathcal{U}_{M}\right)$ for all $t>0$. Fix $t>0$. Then given $n \in \mathbb{N}$ we have that $1_{U_{n}^{M}} \not \leq M_{t}$ (where $\left\{U_{n}^{M} \mid n \in \mathbb{N}\right\}$ is the base for $\mathcal{U}_{M}$ defined in Remark 2.5). In fact, let $x, y \in X$ be two distinct points such that $M\left(x, y, \frac{1}{n}\right)>1-\frac{1}{n}$. Then $1_{U_{n}^{M}}(x, y)=1$ but $M_{t}(x, y)=M(x, y, t)<1$. Consequently, $M_{t} \notin \mathcal{U}_{M}^{01}$ so $\omega\left(\mathcal{U}_{M}\right) \nsubseteq \mathcal{U}_{M}^{01}$.

This also shows that $\mathcal{U}_{M}^{H} \nsubseteq \mathcal{U}_{M}^{01}$.

We notice that even for metric spaces, $\omega\left(\mathcal{U}_{d}\right)$ is not included in $\mathcal{U}_{M_{d}}^{01}$.

Example 4.10. Let $e$ be the euclidean metric on $I$ and define $U: I \times I \rightarrow I$ by

$$
U(x, y)=1-e(x, y), \quad x, y \in X .
$$

Since $U^{-1}((\varepsilon, 1])=\{(x, y) \in I \times I \mid e(x, y)<1-\varepsilon\}=U_{e, 1-\varepsilon} \in \mathcal{U}_{e}$ for each $0<\varepsilon<1$, we have that $U \in \omega\left(\mathcal{U}_{e}\right)$. Nevertheless, $U \notin \mathcal{U}_{M_{e}}^{01}$. Otherwise, we could find $\delta>0$ such that $1_{U_{e, \delta}} \leq U$ where $U_{e, \delta}=\{(x, y) \in I \times I \mid e(x, y)<\delta\}$. However, if $0<e(x, y)<\delta$ then $1_{U_{e, \delta}}(x, y)=1 \not \leq U(x, y)$. Hence $\omega\left(\mathcal{U}_{e}\right) \nsubseteq \mathcal{U}_{M_{e}}^{01}$ (so, by Proposition 4.6, $\left.\omega\left(\mathcal{U}_{e}\right) \nsubseteq \mathcal{U}_{M_{e}}\right)$.

Remark 4.11. As a consequence of the previous examples, we have that if $(X, d)$ is a metric space, the following diagram is not, in general, commutative:

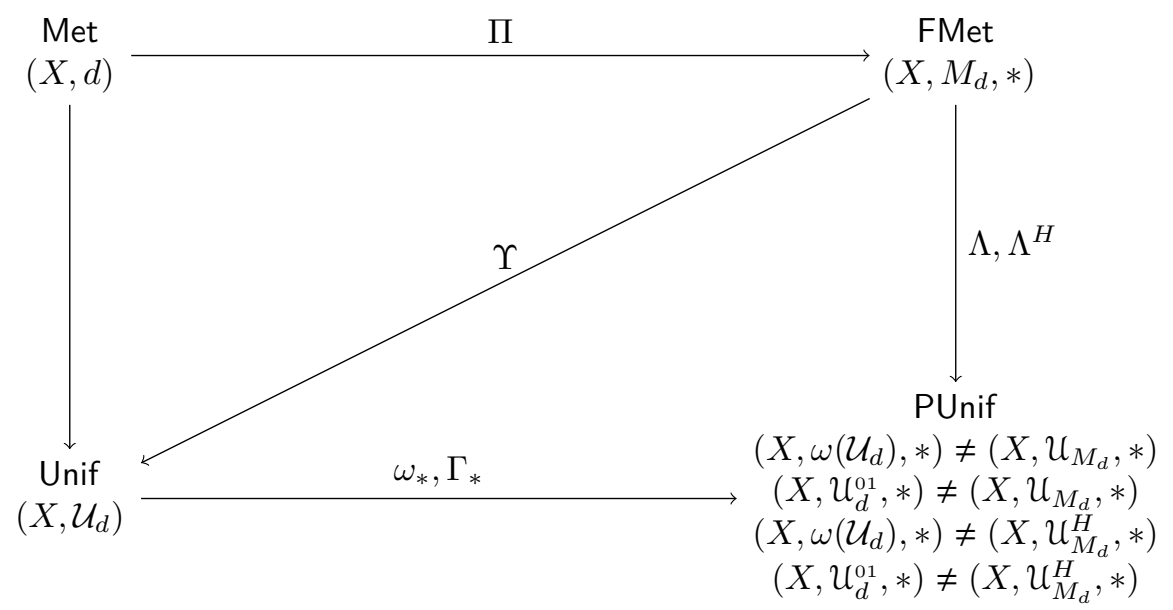


Although a probabilistic uniformity is not necessarily a Lowen uniformity (see Remark 3.9), it is easy to see that the functor $\iota$ of Theorem 3.8 also works for probabilistic uniformities. Thus we can obtain the following:

Proposition 4.12. Let $(X, M, *)$ be a fuzzy metric space. Then:

$$
\mathcal{U}_{M}=\iota\left(\mathcal{U}_{M}\right)=\iota\left(\mathcal{U}_{M}^{H}\right)=\iota\left(\mathcal{U}_{M}^{01}\right)=\iota\left(\omega\left(\mathcal{U}_{M}\right)\right)
$$

Furthermore, if $*$ does not have nontrivial zero divisors then:

$$
\mathcal{U}_{M}=\Theta\left(\mathcal{U}_{M}^{01}\right)=\Theta\left(\omega\left(\mathcal{U}_{M}\right)\right) \text {. }
$$

Proof. We first show that $\mathcal{U}_{M} \subseteq \iota\left(\mathcal{U}_{M}\right)$. Let $n \in \mathbb{N}, U_{n}^{M} \in \mathcal{U}_{M}$ and $\delta \in(0,1)$ such that $(1-\delta) *(1-\delta)>1-\frac{1}{n}$. Then $\left(U_{\delta, \frac{1}{n}}^{M}\right)^{-1}((1-\delta, 1]) \subseteq U_{n}^{M}$. In fact, if $U_{\delta, \frac{1}{n}}^{M}(x, y)=(1-\delta) \rightarrow M\left(x, y, \frac{1}{n}\right)>1-\delta$ then $1-\frac{1}{n}<(1-\delta) *(1-\delta) \leq M\left(x, y, \frac{1}{n}\right)$. Hence $\mathcal{U}_{M} \subseteq \iota\left(\mathcal{U}_{M}\right)$.

On the other hand, by Theorem $3.8(3)$ we have that $\iota\left(\omega\left(\mathcal{U}_{M}\right)\right)=\mathcal{U}_{M}$ so from Proposition 4.6 and the easy fact that $\iota$ is order preserving we deduce the four equalities of the first display.

The equalities of the second display follow from Propositions 3.4 and 3.11.

Remark 4.13. Notice that, in general, $\Theta\left(\mathcal{U}_{M}\right) \neq \mathcal{U}_{M}$ and $\Theta\left(\mathcal{U}_{M}^{H}\right) \neq \mathcal{U}_{M}$ as Example 4.7 shows.

FMet

Unif

PUnif

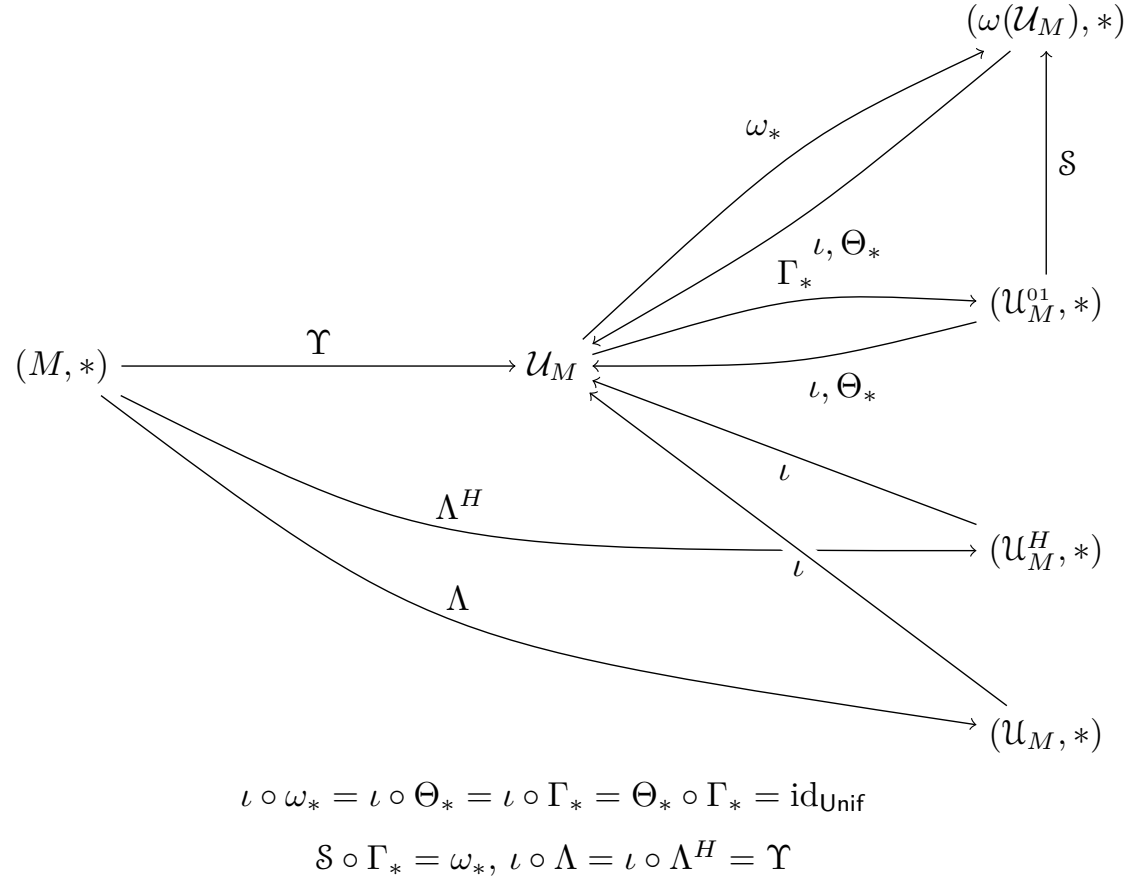

(Recall that when $\Theta_{*}$ acts the $t$-norm $*$ cannot have nontrivial zero divisors.)

In the following, we show that these four probabilistic uniformities have a good behaviour with respect to an important uniform property: precompactness. We recall the following definition. 
Definition $4.14([9])$. A fuzzy metric space $(X, M, *)$ is said to be precompact if for all $t>0$ and $\varepsilon \in(0,1)$ there exists a finite subset $A$ of $X$ such that $X=$ $B_{M}(A, \varepsilon, t)=\bigcup_{a \in A} B_{M}(a, \varepsilon, t)$.

On the other hand, Lowen and Wuyts [29] introduced this concept in the realm of Lowen uniform spaces endowed with the $t$-norm $\wedge$. Nevertheless their definition makes also sense for probabilistic uniform spaces with respect to an arbitrary continuous $t$-norm.

Definition 4.15 (cf. [29, Definition 3.1]). A probabilistic uniform space $(X, \mathcal{U}, *)$ is said to be precompact if for all $U \in \mathcal{U}$ and $\varepsilon \in I_{0}$ there exists a finite subset $A$ of $X$ such that $\bigvee_{a \in A} U(a, x)>1-\varepsilon$ for all $x \in X$. This is equivalent to assert that $X=\bigcup_{a \in A} U(a, \varepsilon)$, where $U(a, \varepsilon)=\{x \in X \mid U(a, x)>1-\varepsilon\}$.

Proposition 4.16. Let $(X, M, *)$ be a fuzzy metric space. The following statements are equivalent:

(1) $(X, M, *)$ is precompact;

(2) $\left(X, \omega\left(\mathcal{U}_{M}\right), *\right)$ is precompact;

(3) $\left(X, \mathcal{U}_{M}^{01}, *\right)$ is precompact;

(4) $\left(X, \mathcal{U}_{M}^{H}, *\right)$ is precompact;

(5) $\left(X, \mathfrak{U}_{M}, *\right)$ is precompact.

Proof. (1) $\Longrightarrow(2)$ : Let $U \in \omega\left(\mathcal{U}_{M}\right)$ and $\varepsilon \in(0,1)$. Since $U^{-1}((1-\varepsilon, 1]) \in \mathcal{U}_{M}$ we can find $n \in \mathbb{N}$ such that $U_{n} \subseteq U^{-1}((1-\varepsilon, 1])$. Furthermore we can find a finite subset $A$ of $X$ with

$$
X=\bigcup_{a \in A} B_{M}\left(a, \frac{1}{n}, \frac{1}{n}\right)=\bigcup_{a \in A} U_{n}(a) \subseteq \bigcup_{a \in A}\left(U^{-1}((1-\varepsilon, 1])\right)(a)=\bigcup_{a \in A} U(a, \varepsilon) .
$$

Therefore, $\left(X, \omega\left(\mathcal{U}_{M}\right), *\right)$ is precompact.

$(2) \Longrightarrow(3) \Longrightarrow(5)$ and $(2) \Longrightarrow(4) \Longrightarrow(5)$ follow from Proposition 4.6.

$(5) \Longrightarrow(1)$ : Let $\varepsilon \in(0,1)$ and $t>0$. Then we can find $\delta \in(0,1)$ such that $1-\varepsilon<(1-\delta) *(1-\delta)$. By assumption there exists a finite subset $A$ of $X$ with $X=\bigcup_{a \in A} U_{\delta, t}(a, \delta)$. Consequently, given $x \in X$ there exists $a \in A$ such that $1-\delta<(1-\delta) \rightarrow M(x, a, t)$ so $M(x, a, t)>(1-\delta) *(1-\delta)>1-\varepsilon$. Therefore, $X=\bigcup_{a \in A} B_{M}(a, \varepsilon, t)$.

\section{Hutton QUASI-UNIFORMity INDUCED By a FUZZY MEtric SPACE}

In the following, we will always consider the unit interval endowed with a continuous $t$-norm $*$ and an order reversing involution, i.e., a unary operation ' $: I \rightarrow I$ such that $\alpha^{\prime \prime}=\alpha$ and $\beta^{\prime} \leq \alpha^{\prime}$ whenever $\alpha \leq \beta$ for all $\alpha, \beta \in I$. Given $a \in I^{X}$, we will denote by $a^{\prime}$ the element of $I^{X}$ given by $a^{\prime}(x)=(a(x))^{\prime}$ for every $x \in X$.

Given a nonempty set $X$, let $\mathcal{H}(X)$ denote the family of all $W: I^{X} \rightarrow I^{X}$ such that:

- $W(a) \geq a$ for all $a \in I^{X}$;

- $W\left(\bigvee_{i \in I} a_{i}\right)=\bigvee_{i \in I} W\left(a_{i}\right)$ for all $\left\{a_{i} \mid i \in I\right\} \subseteq I^{X}$ and $W\left(1_{\varnothing}\right)=1_{\varnothing}$.

Since $a=\bigvee_{x \in X} a(x) * 1_{\{x\}}$ for each $a \in I^{X}$ it follows that every $W \in \mathcal{H}(X)$ is completely determined by the family

$$
\left\{W\left(\alpha * 1_{\{x\}}\right) \mid \alpha \in I_{0}, x \in X\right\} .
$$


Definition $5.1([20])$. Let $X$ be a nonempty set. A Hutton $[0,1]$-quasi-uniformity on $X$ is a nonempty subset $\mathfrak{U}$ of $\mathcal{H}(X)$ such that

(HU1) if $U \in \mathfrak{U}, U \leq V$ and $V \in \mathcal{H}(X)$ then $V \in \mathfrak{H}$;

(HU2) if $U, V \in \mathfrak{U}$ there exists $W \in \mathfrak{U}$ such that $W \leq U$ and $W \leq V$;

(HU3) if $U \in \mathfrak{U}$ there exists $V \in \mathfrak{U}$ such that $V \circ V \leq U$.

If $\mathfrak{U}$ also satisfies

(HU4) if $U \in \mathfrak{U}$ then $U^{-1} \in \mathfrak{U}$

where $U^{-1}(a)=\bigwedge\left\{b \in I^{X} \mid U\left(b^{\prime}\right) \leq a^{\prime}\right\}$, then it is called a Hutton [0,1]-uniformity. In this case, the pair $(X, \mathfrak{U})$ is called a Hutton [0,1]-(quasi-) uniform space.

A function $f:(X, \mathfrak{U}) \rightarrow(Y, \mathfrak{S})$ between two Hutton [0,1]-(quasi-)uniform spaces is said to be uniformly continuous if for every $V \in \mathbb{S}$ there exists $U \in \mathfrak{U}$ such that

$$
U(a) \leq V\left(\bigvee_{x \in X} a(x) * 1_{\{f(x)\}}\right) \circ f, \quad a \in I^{X} .
$$

We denote by $\mathrm{H}(\mathrm{Q})$ Unif the category of Hutton [0,1](-quasi)-uniform spaces and uniformly continuous functions.

Definition 5.2. A base for a Hutton [0,1]-(quasi-)uniformity $\mathfrak{U}$ on $X$ is a nonempty subset $\mathfrak{B}$ of $\mathcal{H}(X)$ such that for each $U \in \mathfrak{U}$ there exists $B \in \mathfrak{B}$ such that $B \leq U$.

If $\mathfrak{B}$ is a nonempty subset of $\mathcal{H}(X)$ verifying:

(1) if $B_{1}, B_{2}, \in \mathfrak{B}$ there exists $B_{3} \in \mathfrak{B}$ such that $B_{3} \leq B_{1}$ and $B_{3} \leq B_{2}$;

(2) if $B_{1} \in \mathfrak{B}$ there exists $B_{2} \in \mathfrak{B}$ such that $B_{2} \circ B_{2} \leq B_{1}$;

then we say that it is a basis for a Hutton $[0,1]$-quasi-uniformity given by

$$
\mathfrak{U}_{\mathfrak{B}}=\{U \in \mathcal{H}(X) \mid \text { there exists } B \in \mathfrak{B} \text { such that } B \leq U\} .
$$

In 1984, Katsaras established the following relations between the categories of (quasi-)uniform spaces and Hutton (quasi-)uniform spaces.

Proposition 5.3 ([22]). Let $(X, \mathcal{U})$ be a (quasi-) uniform space and $(X, \mathfrak{U})$ be a Hutton $[0,1]$-(quasi-) uniform space. Let $\Phi(\mathcal{U})$ be the Hutton [0,1]-(quasi-)uniformity generated by

$$
\{\phi(U) \mid U \in \mathcal{U}\}
$$

where $\phi(U)(a)(y)=\bigvee_{x \in X} a(x) * 1_{U(x)}(y)=\bigvee_{y \in U(x)} a(x)$ for each $U \in \mathcal{U}, a \in I^{X}$ and $y \in X$. Let $\Psi(\mathfrak{H})$ be the (quasi-) uniformity generated by

$$
\{\psi(U) \mid U \in \mathfrak{H}\}
$$

where $\psi(U)=\left\{(x, y) \in X \times X \mid \forall a \in I^{X}, a(x) \leq U(a)(y)\right\}$ for each $U \in \mathfrak{U}$. Then:

(1) $\Phi:(\mathrm{Q})$ Unif $\rightarrow \mathrm{H}(\mathrm{Q})$ Unif is a functor sending each $(X, \mathcal{U})$ to $(X, \Phi(\mathcal{U}))$ and leaving morphisms unchanged;

(2) $\Psi: \mathrm{H}(\mathrm{Q})$ Unif $\rightarrow(\mathrm{Q})$ Unif is a functor sending each $(X, \mathfrak{H})$ to $(X, \Psi(\mathfrak{H}))$ and leaving morphisms unchanged;

(3) $\Psi(\Phi(\mathcal{U}))=\mathcal{U}$

(4) $\mathfrak{U} \subseteq \Phi(\Psi(\mathfrak{U}))$;

(5) $\Psi$ is a right adjoint of $\Phi$.

On the other hand, Höhle [16, p. 313] showed how to construct a Hutton uniformity by means of a probabilistic uniformity as follows: 
Proposition $5.4([16])$. Let $*$ be a continuous $t$-norm such that the unary operation ' on I given by $a^{\prime}:=a \stackrel{*}{\rightarrow} 0=\bigvee\{b \in[0,1] \mid a * b=0\}$ for each $a \in I$ is an order reversing involution. Let $(\mathcal{U}, *)$ be a probabilistic uniformity on $X$. Then the family $\left\{W_{U} \mid U \in \mathcal{U}\right\}$ is a base for a Hutton $[0,1]$-uniformity $\Xi(\mathcal{U})$ on $X$, where $W_{U} \in\left(I^{X}\right)^{I^{X}}$ is given by

$$
W_{U}(a)(y)=\bigvee_{x \in X} a(x) * U(x, y) \quad a \in I^{X}, y \in X
$$

Remark 5.5. Note that if $*$ is such that $a^{\prime \prime} \neq a$ for some $a \in I$, then $\Xi(\mathcal{U})$ is, in general, a Hutton [0,1]-quasi-uniformity instead of a Hutton [0,1]-uniformity.

Proposition 5.6. Let $*$ be a continuous t-norm such that the unary operation ' on I given by $a^{\prime}:=a \stackrel{*}{\rightarrow} 0=\bigvee\{b \in[0,1] \mid a * b=0\}$ for each $a \in I$ is an order reversing involution. The map $\Xi:$ PUnif $\rightarrow$ HUnif sending $(X, \mathcal{U}, *)$ to $(X, \Xi(\mathcal{U}))$ and leaving morphisms unchanged is a fully faithful functor.

Proof. By Proposition 5.4 we already know that $\Xi(\mathcal{U})$ is a Hutton $[0,1]$-uniformity. Furthermore, let $(X, \mathcal{U}, *),(Y, \mathcal{V}, \star)$ be two probabilistic uniform spaces. Then $f:(X, \Xi(\mathcal{U})) \rightarrow(Y, \Xi(\mathcal{V}))$ is uniformly continuous if for every $V \in \mathcal{V}$ there exists $U \in \mathcal{U}$ such that $W_{U}(a) \leq W_{V}\left(\bigvee_{x \in X} a(x) * 1_{\{f(x)\}}\right) \circ f$ for each $a \in I^{X}$. But

$$
\begin{aligned}
& \forall a \in I^{X} \quad W_{U}(a) \leq W_{V}\left(\bigvee_{x \in X} a(x) * 1_{\{f(x)\}}\right) \circ f \\
\Longleftrightarrow & \forall x \in X, \forall \alpha \in I_{0} \quad W_{U}\left(\alpha * 1_{\{x\}}\right) \leq W_{V}\left(\alpha * 1_{\{f(x)\}}\right) \circ f \\
\Longleftrightarrow & \forall x, y \in X, \forall \alpha \in I_{0} \quad \alpha * U(x, y) \leq \alpha * V(f(x), f(y)) \\
\Longleftrightarrow & \forall x, y \in X \quad U(x, y) \leq V(f(x), f(y))
\end{aligned}
$$

and we conclude that $f:(X, \Xi(\mathcal{U})) \rightarrow(Y, \Xi(\mathcal{V}))$ is uniformly continuous if and only if $f:(X, \mathcal{U}, *) \rightarrow(Y, \mathcal{V}, \star)$ is uniformly continuous.

Remark 5.7. Observe that the above proposition is a special case of [43, Proposition 4.2$]$.

In [12] the authors developed a method to construct a Hutton [0, 1]-quasi-uniformity from a fuzzy metric space as follows.

Proposition 5.8 ([12, Proposition 14]). Let (X, M,*) be a fuzzy (quasi-)metric space. Then the family $\mathfrak{B}_{M}=\left\{W_{\varepsilon, t}^{M} \mid \varepsilon \in I_{0}, t>0\right\}$ is a base for a Hutton $[0,1]$-quasi-uniformity $\mathfrak{H}_{M}$ on $X$ where $W_{\varepsilon, t}^{M}: I^{X} \rightarrow I^{X}$ is given by

$$
W_{\varepsilon, t}^{M}(a)(y)=\bigvee_{x \in X} a(x) *((1-\varepsilon) \rightarrow M(x, y, t)), \quad a \in I^{X}, y \in X
$$

This construction gives a factorization via Hutton [0,1]-quasi-uniformities of the association of a uniformity to each fuzzy metric space (see Remark 5.15).

Later on, Yue and Fang [41] provided another construction of a Hutton [0,1]quasi-uniformity for fuzzy metric spaces in the sense of Kramosil and Michalek which is also valid in our context.

Proposition 5.9 ([41, Section 5$])$. Let $(X, M, *)$ be a fuzzy metric space. Then the family $\mathfrak{B}^{M}=\left\{W_{t}^{M} \mid t>0\right\}$ is a base for a Hutton $[0,1]$-quasi-uniformity $\mathfrak{H}_{M}^{H}$ on $X$ where $W_{t}^{M}: I^{X} \rightarrow I^{X}$ is given by

$$
W_{t}^{M}(a)(y)=\bigvee_{x \in X} a(x) * M(x, y, t), \quad a \in I^{X}, y \in X
$$


On the other hand, in Section 3 we have considered two functors $\Gamma_{*}$ and $\omega_{*}$ and in Section 4 two mappings $\Lambda^{H}$ and $\Lambda$ which allow to obtain probabilistic uniformities by means of uniformities and fuzzy metrics respectively. Then we can compose these mappings with the functor $\Xi$ in order to obtain

$$
\Xi \circ \omega_{*}, \Xi \circ \Gamma_{*} \text { : Unif } \rightarrow \text { PUnif and } \Xi \circ \Lambda, \Xi \circ \Lambda^{H}: \operatorname{obj}(\text { FMetric) } \rightarrow \operatorname{obj}(\text { PUnif). }
$$

This provides four methods of generating a Hutton [0,1]-quasi-uniformity from a fuzzy metric space that we will study next. It is obvious that the first two mappings are functors but the last two are not since $\Lambda$ and $\Lambda^{H}$ are not functors (see Remark 4.2). We first observe that given a fuzzy metric space $(X, M$, *), the images of $\mathcal{U}_{M}$ under $\Xi \circ \Lambda$ and $\Xi \circ \Lambda^{H}$ are, respectively, $\mathfrak{U}_{M}$ (see Proposition 5.8) and $\mathfrak{U}_{M}^{H}$ (see Proposition 5.9).

Proposition 5.10. Let $(X, M, *)$ be a fuzzy metric space. Then:

$$
(\Xi \circ \Lambda)\left(\mathcal{U}_{M}\right)=\Xi\left(\mathcal{U}_{M}\right)=\mathfrak{U}_{M}, \quad \text { and } \quad\left(\Xi \circ \Lambda^{H}\right)\left(\mathcal{U}_{M}\right)=\Xi\left(\mathcal{U}_{M}^{H}\right)=\mathfrak{U}_{M}^{H} .
$$

Proof. Given $\varepsilon \in I_{0}$ and $t>0$, let $U_{\varepsilon, t}^{M}$ be a member of the basis of $\mathcal{U}_{M}$ as defined in Proposition 4.1. By Proposition 5.4 we have that

$W_{U_{\varepsilon, t}^{M}}(a)(y)=\bigvee_{x \in X} a(x) * U_{\varepsilon, t}^{M}(x, y)=\bigvee_{x \in X} a(x) *((1-\varepsilon) \rightarrow M(x, y, t))=W_{\varepsilon, t}^{M}(a)(y)$ so $\Xi\left(\mathcal{U}_{M}\right)=\mathfrak{U}_{M}$. In a similar way you can obtain that $\Xi\left(\mathfrak{U}_{M}^{H}\right)=\mathfrak{U}_{M}^{H}$.

We present now an example showing that $\Xi \circ \Lambda$ is not a functor when leaving morphisms unchanged, as it was incorrectly stated in [12, Proposition 17].

Example 5.11 (cf. Remark 4.2). Let $e$ be the euclidean metric on $\mathbb{R}$ and $*_{t}$ be the Łukasiewicz $t$-norm. Let us consider the fuzzy metric spaces $\left(\mathbb{R}, N, *_{七}\right)$ and $\left(\mathbb{R}, M_{e}, *\right)$, where $N$ is the fuzzy set given by $N(x, y, t)=\max \left\{M_{e}(x, y, t), \frac{1}{2}\right\}$. We proved in Remark 4.2 that the identity function id: $\left(\mathbb{R}, N, *_{€}\right) \rightarrow\left(\mathbb{R}, M_{e}, *_{€}\right)$ is uniformly continuous.

Nevertheless, id: $\left(\mathbb{R}, \mathfrak{U}_{N}\right) \rightarrow\left(\mathbb{R}, \mathfrak{U}_{M_{e}}\right)$ is not uniformly continuous. Indeed, let $\varepsilon \in(0,1 / 2)$ and $t>0$. It is obvious that there exist $x, y \in \mathbb{R}$ such that $M_{e}(x, y, t)+$ $\varepsilon<\frac{1}{2}$ so $M_{e}(x, y, t)<1-\varepsilon$ which implies $W_{\varepsilon, t}^{M_{e}}\left(1_{\{x\}}\right)(y)=M_{e}(x, y, t)+\varepsilon$. Then for each $\delta \in I_{0}$ and $s>0$ we have that

$$
W_{\varepsilon, t}^{M_{e}}\left(1_{\{x\}}\right)(y)=M_{e}(x, y, t)+\varepsilon<\frac{1}{2} \leq N(x, y, s) \leq W_{\delta, s}^{N}\left(1_{\{x\}}\right)(y) .
$$

Hence $W_{\delta, s}^{N} \not \leq W_{\varepsilon, t}^{M_{e}}$ for every $\delta \in I_{0}$ and $s>0$ so id: $\left(\mathbb{R}, \mathfrak{U}_{N}\right) \rightarrow\left(\mathbb{R}, \mathfrak{U}_{M_{e}}\right)$ is not uniformly continuous.

Definition 5.12. Let $(X, \mathcal{U})$ be a uniform space and $\left(\mathcal{U}^{01}, *\right)$ be the probabilistic uniformity of Notation 4.4. Then the family $\left\{W_{1_{U}} \mid U \in \mathcal{U}\right\}$ is a base for a Hutton quasi-uniformity $\mathfrak{U}^{01}:=\Xi\left(\mathcal{U}^{01}\right)=\left(\Xi \circ \Gamma_{*}\right)(\mathcal{U})$ where

$$
W_{1_{U}}(a)(y)=\bigvee_{x \in X} a(x) * 1_{U}(x, y)=\bigvee_{y \in U(x)} a(x), \quad a \in I^{X}, y \in X
$$

Definition 5.13. Let $(X, \mathcal{U})$ be a uniform space and $\omega(\mathcal{U})$ be the Lowen uniformity generated by $\mathcal{U}$. Then the family $\left\{W_{U} \mid U \in \omega(\mathcal{U})\right\}$ is a base for a Hutton [0,1]quasi-uniformity $\mathfrak{U}^{\omega}:=(\Xi \circ \omega)(\mathcal{U})$ where

$$
W_{U}(a)(y)=\bigvee_{x \in X} a(x) * U(x, y), \quad U \in \omega(\mathcal{U}), a \in I^{X}, y \in X
$$


Given a fuzzy metric space $(X, M, *)$, we know from Proposition 4.6 that $\mathfrak{U}_{M} \subseteq$ $\mathcal{U}_{M}^{01} \wedge \mathcal{U}_{M}^{H} \subseteq \mathcal{U}_{M}^{01} \vee \mathcal{U}_{M}^{H} \subseteq \omega\left(\mathcal{U}_{M}\right)$. Consequently, a similar relationship is obtained for their associated Hutton [0,1]-quasi-uniformities:

$$
\mathfrak{U}_{M} \subseteq \mathfrak{U}_{M}^{01} \wedge \mathfrak{U}_{M}^{H} \subseteq \mathfrak{U}_{M}^{01} \vee \mathfrak{U}_{M}^{H} \subseteq \mathfrak{U}_{M}^{\omega} .
$$

In [41, Example 5.1] it is proved that, in general, $\mathfrak{H}_{M}^{H} \nsubseteq \mathfrak{U}_{M}$. We provide here other examples showing that the other inclusions are, in general, strict.

Example 5.14. Let $e$ be the euclidean metric on $\mathbb{R}$ and $*$ be the product $t$-norm. We show that $\mathfrak{U}_{M_{e}}^{01} \nsubseteq \Xi\left(\mathcal{U}_{M_{e}}\right)=\mathfrak{U}_{M_{e}}$. Let $a \in I^{\mathbb{R}}$ be the characteristic function of $\mathbb{R} \backslash[-1,1]$. Given $U=\left\{(x, y) \in \mathbb{R}^{2} \mid e(x, y)<1\right\} \in \mathcal{U}_{e}=\mathcal{U}_{M_{e}}$ we have $W_{1_{U}}(a)(0)=\bigvee_{0 \in U(x)} a(x)=0<W_{\varepsilon, t}^{M_{e}}(a)(0) \wedge W_{t}^{M_{e}}(a)(0)$ for each $\varepsilon \in I_{0}$ and $t>0$ and so $W_{\varepsilon, t}^{M_{e}} \not \leq W_{1_{U}}$ and $W_{t}^{M_{e}} \not \leq W_{1_{U}}$, i.e. $\mathfrak{U}_{M_{e}}^{0_{1}} \nsubseteq \mathfrak{U}_{M_{e}}$ and $\mathfrak{H}_{M_{e}}^{01} \nsubseteq \mathfrak{H}_{M_{e}}^{H}$.

On the other hand, given $t>0$ then $M_{e, t} \in \mathcal{U}_{M_{e}}^{H}$. For each $n \in \mathbb{N}$ let $a_{n} \in I^{\mathbb{R}}$ be given by

$$
a_{n}(x)= \begin{cases}1, & \text { if } x=\frac{1}{2 n(n-1)} \\ 0, & \text { otherwise }\end{cases}
$$

It is easy to check that $M_{e}\left(0, \frac{1}{2 n(n-1)}, \frac{1}{n}\right)>1-\frac{1}{n}$. Then we have that $W_{t}^{M_{e}}\left(a_{n}\right)(0)<$ $1=W_{1_{U_{n}}}\left(a_{n}\right)(0)$. Therefore, $W_{1_{U_{n}}} \not W_{t}^{M_{e}}$ for all $n \in \mathbb{N}$ so $\mathfrak{U}_{M_{e}}^{H} \nsubseteq \mathfrak{U}_{M_{e}}^{01}$. This also shows that $\mathfrak{U}_{M_{e}}^{\omega} \nsubseteq \mathfrak{U}_{M_{e}}^{01}$.

Remark 5.15. In [12, Corollary 16] it was proved that $\Psi\left(\mathfrak{U}_{M}\right)=\mathcal{U}_{M}$ for a fuzzy metric space $(X, M, *)$. Consequently, the following diagram commutes:

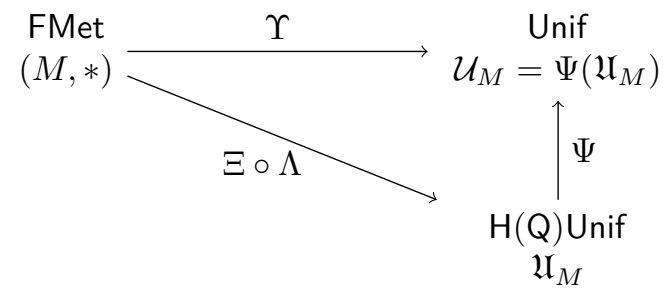

It is natural to wonder whether $\Psi\left(\mathfrak{U}_{M}^{01}\right)=\mathcal{U}_{M}, \Psi\left(\mathfrak{U}_{M}^{H}\right)=\mathcal{U}_{M}$ or $\Psi\left(\mathfrak{U}_{M}^{\omega}\right)=\mathcal{U}_{M}$. We solve this question in the following.

Proposition 5.16.

(1) Katsaras's functor $\Phi$ can be factorized as $\Phi=\Xi \circ \Gamma_{*}$.

(2) $\Psi \circ \Xi \circ \Gamma_{*}=1_{\text {Unif }}$.

Consequently, $\Phi(\mathcal{U})=(\Xi \circ \Gamma)(\mathcal{U})=\mathfrak{l}^{01}, \Psi\left(\mathfrak{U}^{01}\right)=\mathcal{U}$ and the following diagram commutes:

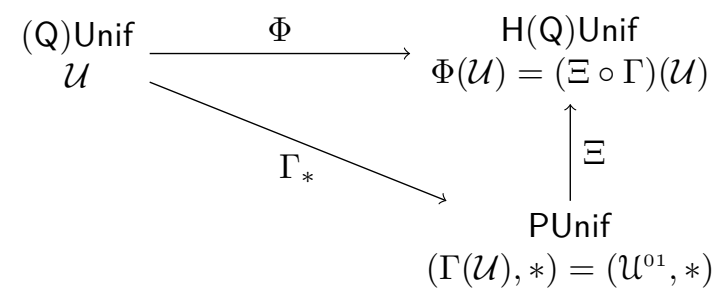

Proof. (1) follows immediately from the definition of $\Phi$ and Definition 5.12 and (2) follows from (1) and Proposition 5.3 (4). 
We observe that the above result gives a factorization of the Katsaras functor $\Phi$ via probabilistic uniform spaces by means of the functors $\Gamma_{*}$ and $\Xi$.

Remark 5.17. Nevertheless, given a fuzzy metric space $(X, M, *)$ in general $\Psi\left(\mathfrak{U}_{M}^{\omega}\right) \neq$ $\mathcal{U}_{M}$ and $\Psi\left(\mathfrak{U}_{M}^{H}\right) \neq \mathcal{U}_{M}$. Let us consider the fuzzy metric space $\left(\mathbb{R}, M_{e}, \cdot\right)$ where $e$ is the euclidean metric. Then we have that $M_{e, t} \in \omega\left(\mathcal{U}_{M_{e}}\right) \cap \mathcal{U}_{M_{e}}^{H}$ where $M_{e, t}(x, y)=$ $M_{e}(x, y, t)$ for all $t>0$ and $x, y \in \mathbb{R}$. Then we have

$$
\begin{aligned}
\psi\left(W_{M_{t}}\right) & =\left\{(x, y) \in \mathbb{R} \times \mathbb{R} \mid \forall a \in I^{\mathbb{R}}, a(x) \leq W_{M_{t}}(a)(y)\right\} \\
& =\left\{(x, y) \in \mathbb{R} \times \mathbb{R} \mid \forall \alpha \in I_{0}, \forall z \in \mathbb{R},\left(\alpha \cdot 1_{\{z\}}\right)(x) \leq W_{M_{t}}\left(\alpha \cdot 1_{\{z\}}\right)(y)\right\} \\
& =\left\{(x, y) \in \mathbb{R} \times \mathbb{R} \mid \forall \alpha \in I_{0}, \alpha \leq W_{M_{t}}\left(\alpha \cdot 1_{\{x\}}\right)(y)=\alpha \cdot M_{t}(x, y)\right\} \\
& =\{(x, y) \in \mathbb{R} \times \mathbb{R} \mid M(x, y, t)=1\}=\{(x, x) \mid x \in \mathbb{R}\} .
\end{aligned}
$$

Consequently, $\Psi\left(\mathfrak{U}_{M_{e}}^{\omega}\right)=\Psi\left(\mathfrak{U}_{M_{e}}^{H}\right)$ is the discrete uniformity which is different from $\mathcal{U}_{M_{e}}=\mathcal{U}_{e}$.

Remark 5.18. As a consequence of our results, the following diagrams commute:
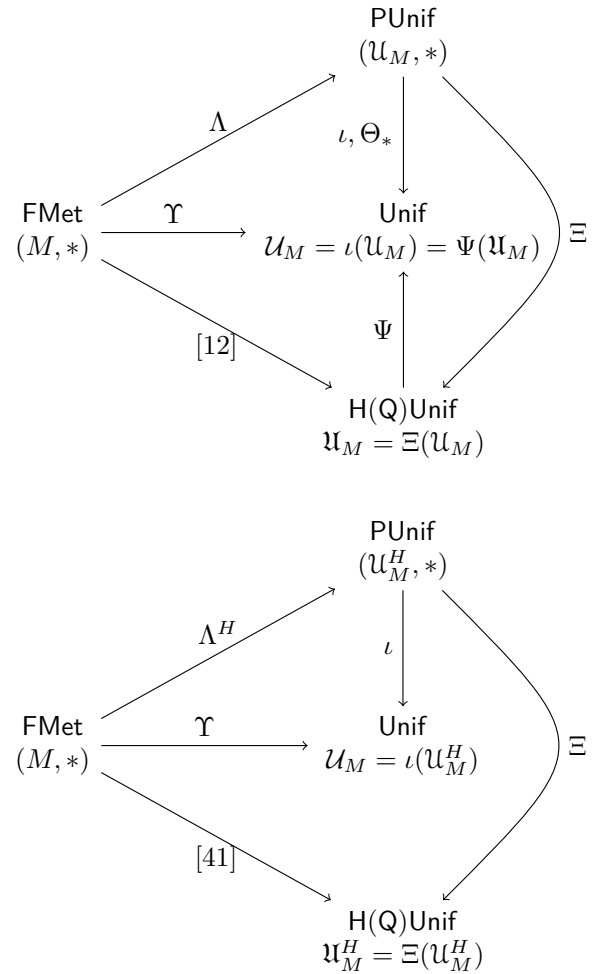
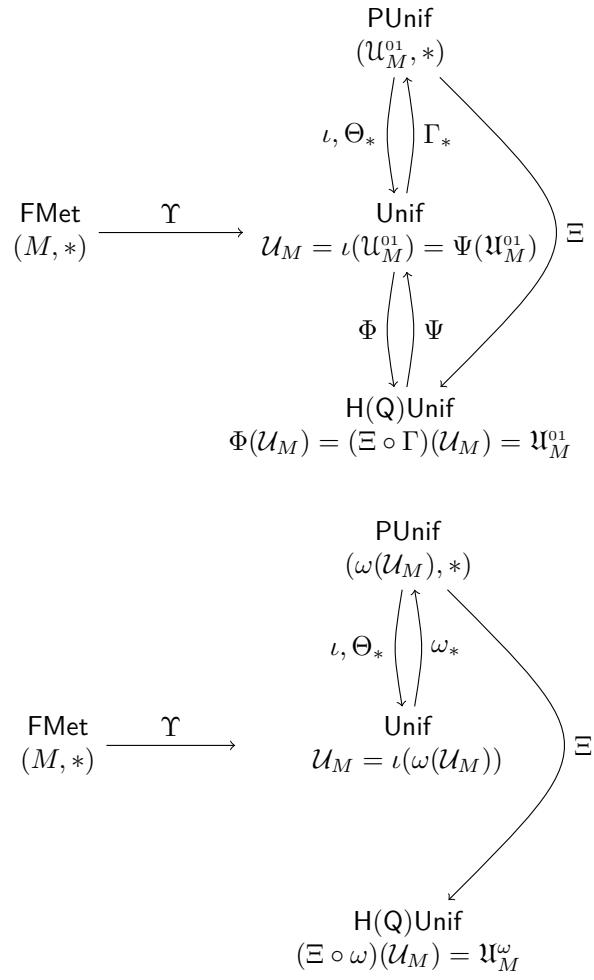

(Recall that when $\Theta_{*}$ acts we need that the $t$-norm $*$ does not have nontrivial zero divisors.)

\section{TOPOlOGIES AND I-TOPOLOGIES GENERATED BY FUZZY UNIFORMITIES}

Finally, we study the classical topologies and the $I$-topologies associated with the fuzzy uniformities that we have considered. We first recall some pertinent definitions. 
Definition 6.1 ([3]). A (Chang) I-topology on a nonempty set $X$ is a subset $\mathfrak{I}$ of $I^{X}$ such that:

(1) $1_{\varnothing}, 1_{X} \in \mathfrak{I}$;

(2) $a \wedge b \in \mathfrak{I}$ whenever $a, b \in \mathfrak{I}$

(3) $\bigvee_{i \in \Lambda} a_{i} \in \mathfrak{I}$ whenever $\left\{a_{i} \mid i \in \Lambda\right\} \subseteq \mathfrak{I}$.

In this case, the pair $(X, \mathfrak{I})$ is called an $I$-topological space.

A function $f:\left(X, \mathfrak{I}_{X}\right) \rightarrow\left(Y, \mathfrak{I}_{Y}\right)$ is continuous if $f^{-1}(a) \in \mathfrak{I}_{X}$ for each $a \in \mathfrak{I}_{Y}$ (where $f^{-1}(a):=a \circ f$ ).

We denote by ITop the category of $I$-topological spaces and continuous functions.

Remark 6.2. By identifying, as usual, subsets of a given set with the corresponding characteristic functions we can treat a topological space $(X, \tau)$ as an $I$-topological space. So we have a functor $\Gamma_{t}$ : Top $\rightarrow$ ITop which identifies the category of topological spaces with the full subcategory of ITop whose objects are just those $I$-topological spaces $(X, \mathfrak{I})$ such that $\mathfrak{I} \subseteq 2^{X}$.

With Chang's definition constant maps are not necessarily continuous. This is the reason why Lowen proposed the following:

Definition 6.3 ([25]). A stratified I-topology on a nonempty set $X$ is a subset $\mathfrak{I}$ of $I^{X}$ such that:

(1) $\alpha 1_{X} \in \mathfrak{I}$ for all $\alpha \in I$;

(2) $a \wedge b \in \mathfrak{I}$ for all $a, b \in \mathfrak{I}$;

(3) $\bigvee_{i \in \Lambda} a_{i} \in \mathfrak{I}$ whenever $\left\{a_{i} \mid i \in \Lambda\right\} \subseteq \mathfrak{I}$.

In this case, the pair $(X, \mathfrak{I})$ is called a stratified $I$-topological space.

A function $f:\left(X, \mathfrak{I}_{X}\right) \rightarrow\left(Y, \mathfrak{I}_{Y}\right)$ is continuous if $f^{-1}(a) \in \mathfrak{I}_{X}$ for each $a \in \mathfrak{I}_{Y}$.

We denote by SITop the category of stratified $I$-topological spaces and continuous functions.

Definition 6.4 ([25]). An operator $\psi: I^{X} \rightarrow I^{X}$ is a fuzzy closure operator if

(1) $\psi(\alpha)=\alpha$ for all constant $\alpha \in I^{X}$;

(2) $a \leq \psi(a)$ for all $a \in I^{X}$;

(3) $\psi(a \vee b)=\psi(a) \vee \psi(b)$ for all $a, b \in I^{X}$;

(4) $\psi(\psi(a))=\psi(a)$ for all $a \in I^{X}$.

In [25], Lowen introduced two functors to obtain a relationship between stratified $I$-topologies and classical topologies as follows:

Proposition 6.5 ([25]). Let $X$ be a nonempty set, $\tau$ be a topology on $X$ and $\mathfrak{I}$ be a stratified I-topology on $X$. Define

$$
\begin{aligned}
\omega(\tau) & =\left\{a \in I^{X} \mid a \text { is lower semicontinuous }\right\} \quad \text { and } \\
\iota(\mathfrak{I}) & =\left\{a^{-1}((\varepsilon, 1]) \mid \varepsilon \in[0,1), a \in \mathfrak{I}\right\} .
\end{aligned}
$$

Then:

(1) $\omega(\tau)$ is a stratified I-topology;

(2) $\iota(\mathfrak{I})$ is a topology on $X$;

(3) $\iota(\omega(\tau))=\tau$;

(4) $\mathfrak{I} \subseteq \omega(\iota(\mathfrak{I}))$.

Furthermore, if we consider the mappings $\omega:$ Top $\rightarrow$ SITop and $\iota:$ SITop $\rightarrow$ Top, which leave morphisms unchanged, and $\omega((X, \tau))=(X, \omega(\tau))$ and $\iota((X, \mathfrak{I}))=$ $(X, \iota(\mathfrak{I}))$ then they are fully faithful and faithful functors respectively. 
Remark 6.6. We notice that if $(X, \tau)$ is a topological space then $a \in I^{X}$ is $\omega(\tau)$ closed if and only if it is upper semicontinuous, i.e. if

$$
a(x)=\bigwedge_{V \in \mathcal{N}(x)} \bigvee_{y \in V} a(y)
$$

for each $x \in X$, where $\mathcal{N}(x)$ is the neighborhood filter of $x$.

Remark 6.7 (Cf. Proposition 3.10). We can consider a mapping $\mathcal{S}$ : ITop $\rightarrow$ SITop which associates to each $I$-topology $\mathfrak{I}$ the stratified $I$-topology generated by the family $\mathfrak{I} \cup\left\{\alpha 1_{X} \mid \alpha \in I\right\}$ and which leaves morphisms unchanged. It can easily be verified that the continuity of a map $f:\left(X, \mathfrak{I}_{X}\right) \rightarrow\left(Y, \mathfrak{I}_{Y}\right)$ implies the continuity of the map $f:\left(X,\left\langle\mathfrak{I}_{X} \cup\left\{\alpha 1_{X} \mid \alpha \in I\right\}\right\rangle\right) \rightarrow\left(Y,\left\langle\mathfrak{I}_{Y} \cup\left\{\alpha 1_{Y} \mid \alpha \in I\right\}\right\rangle\right)$ so $\mathcal{S}$ is a functor.

Then we have the following commutative diagram:

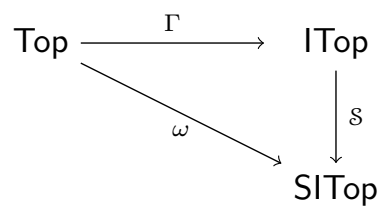

In fact given a topological space $(X, \tau)$ we have that

$$
\begin{aligned}
\mathcal{S}(\Gamma(\tau)) & =\mathcal{S}\left(\left\{1_{G} \mid G \in \tau\right\}\right)=\left\langle\left\{1_{G} \mid G \in \tau\right\} \cup\left\{\alpha 1_{X} \mid \alpha \in I\right\}\right\rangle \\
& =\left\langle\left\{\alpha 1_{G} \mid \alpha \in I \text { and } G \in \tau\right\}\right\rangle=\omega(\tau) .
\end{aligned}
$$

(The last equality follows since given $a \in I^{X}$ we always have $a=\bigvee_{\alpha \in I} \alpha 1_{a^{-1}((\alpha, 1])}$. Hence if $a$ is lower semicontinuous then $a^{-1}((\alpha, 1]) \in \tau$ for each $\alpha \in[0,1]$ and so $a \in\left\langle\left\{\alpha 1_{G} \mid \alpha \in[0,1]\right.\right.$ and $\left.\left.\left.G \in \tau\right\}\right\rangle\right)$.

Any probabilistic uniformity $(\mathcal{U}, *)$ on $X$ induces a stratified $I$-topology $\mathfrak{I}(\mathcal{U})$ by means of the following fuzzy closure operator $\psi_{\mathfrak{I}(u)}: I^{X} \rightarrow I^{X}$ given by (cf. $[27$, Proposition 2.3], [42, p. 411]):

$$
\psi_{\mathfrak{I}(\mathcal{U})}(a)(x)=\bigwedge_{U \in \mathcal{U}} \bigvee_{y \in X} a(y) * U(y, x), \quad x \in X, a \in I^{X} .
$$

(We will omit the subscript if no confusion arises).

Furthermore, Lowen proved the following:

Theorem $6.8([27$, Theorem 3.1$])$. Let $(X, \mathcal{U})$ be a uniform space and $(X, \mathcal{U}, *)$ be a Lowen uniform space. Then:

(1) $\mathfrak{I}(\omega(\mathcal{U}))=\omega(\tau(\mathcal{U}))$;

(2) $\tau(\iota(\mathcal{U}))=\iota(\mathfrak{I}(\mathcal{U}))$.

On the other hand, if $\mathfrak{U}$ is a Hutton [0,1](-quasi)-uniformity then it generates a stratified $I$-topology given by

$$
\mathfrak{I}(\mathfrak{U})=\left\{a \in I^{X} \mid a \leq \bigvee\left\{b \in I^{X} \mid \text { there exists } U \in \mathfrak{U} \text { such that } U(b) \leq a\right\}\right\} .
$$

Remark 6.9. We notice that if $(X, \mathcal{U}, *)$ is a probabilistic uniform space then

$$
\mathfrak{I}(\mathcal{U})=\mathfrak{I}(\Xi(\mathcal{U})) \text {. }
$$

This is a direct consequence of the definition of the two stratified $I$-topologies above and the definition of $\Xi$. 
Now, let $(X, \mathcal{U}, *)$ be a probabilistic uniform space. Then $\tau(\iota(\mathcal{U}))$ is a topology on $X$ which has as a base the family $\left\{U(x, \varepsilon) \mid x \in X, \varepsilon \in I_{0}, U \in \mathcal{U}\right\}$, where $U(x, \varepsilon)=\{y \in X \mid U(x, y)>1-\varepsilon\}$.

Then we have the following result:

Theorem 6.10. Let $(X, M, *)$ be a fuzzy metric space. Then

$$
\begin{aligned}
\tau(M) & =\tau\left(\iota\left(\mathfrak{U}_{M}\right)\right)=\tau\left(\iota\left(\mathfrak{U}_{M}^{01}\right)\right)=\tau\left(\iota\left(\mathfrak{U}_{M}^{H}\right)\right)=\tau\left(\iota\left(\omega\left(\mathcal{U}_{M}\right)\right)\right) \\
& =\tau\left(\Psi\left(\mathfrak{U}_{M}\right)\right)=\tau\left(\Psi\left(\mathfrak{U}_{M}^{01}\right)\right) \\
& =\iota\left(\mathfrak{I}\left(\mathfrak{U}_{M}\right)\right)=\iota\left(\mathfrak{I}\left(\mathfrak{U}_{M}^{01}\right)\right)=\iota\left(\mathfrak{I}\left(\mathfrak{U}_{M}^{H}\right)\right)=\iota\left(\mathfrak{I}\left(\omega\left(\mathcal{U}_{M}\right)\right)\right) \\
& =\iota\left(\mathfrak{I}\left(\mathfrak{U}_{M}\right)\right)=\iota\left(\mathfrak{I}\left(\mathfrak{U}_{M}^{01}\right)\right)=\iota\left(\mathfrak{I}\left(\mathfrak{U}_{M}^{H}\right)\right)=\iota\left(\mathfrak{I}\left(\mathfrak{U}_{M}^{\omega}\right)\right) .
\end{aligned}
$$

Proof. By Proposition 4.12 we have that

$$
\iota\left(\mathcal{U}_{M}\right)=\iota\left(\mathcal{U}_{M}^{01}\right)=\iota\left(\mathcal{U}_{M}^{H}\right)=\iota\left(\omega\left(\mathcal{U}_{M}\right)\right)=\mathcal{U}_{M}
$$

so the first line of equalities is verified. For the second line, we know from Proposition 5.16 that $\Psi\left(\mathfrak{U}_{M}^{01}\right)=\mathcal{U}_{M}$ so $\tau\left(\Psi\left(\mathfrak{H}_{M}^{01}\right)\right)=\tau\left(\mathcal{U}_{M}\right)=\tau(M)$ and by [12, Proposition 22] we obtain that $\tau\left(\Psi\left(\mathfrak{H}_{M}\right)\right)=\tau(M)$. The third line is a consequence of Theorem 6.8. The last chain of equalities is deduced from the previous remark.

Remark 6.11. We notice that, in general, $\tau\left(\Psi\left(\mathfrak{l}_{M}^{\omega}\right)\right) \neq \tau(M)$ and $\tau\left(\Psi\left(\mathfrak{U}_{M}^{H}\right)\right) \neq \tau(M)$ as Remark 5.17 shows.

Now we center our attention in the stratified $I$-topologies generated by the the fuzzy uniformities that we have considered.

Theorem 6.12 ([22, Theorem 2.4]). Let $(X, \mathcal{U})$ be a quasi-uniform space. Then

$$
\mathfrak{I}(\Phi(\mathcal{U}))=\omega(\tau(\mathcal{U})) .
$$

Proposition 6.13. Let $(X, M, *)$ be a fuzzy metric space. Then

$$
\omega\left(\tau\left(\mathcal{U}_{M}\right)\right)=\mathfrak{I}\left(\mathfrak{U}_{M}^{01}\right)=\mathfrak{I}\left(\omega\left(\mathcal{U}_{M}\right)\right)=\mathfrak{I}\left(\mathfrak{H}_{M}^{01}\right)=\mathfrak{I}\left(\mathfrak{U}_{M}^{\omega}\right) .
$$

Proof. Let $a \in I^{X}$ and $x \in X$. Then

$$
\psi_{\mathfrak{I}\left(\mathcal{U}_{M}^{01}\right)}(a)(x)=\bigwedge_{U \in \mathcal{U}_{M}} \bigvee_{y \in X} a(y) * 1_{U}(y, x)=\bigwedge_{U \in \mathcal{U}_{M}} \bigvee_{y \in U^{-1}(x)} a(x)=\psi_{\omega\left(\tau\left(\mathcal{U}_{M}\right)\right)}(a)(x)
$$

so $\omega\left(\tau\left(\mathcal{U}_{M}\right)\right)=\mathfrak{I}\left(\mathcal{U}_{M}^{01}\right)$. Furthermore, $\omega\left(\tau\left(\mathcal{U}_{M}\right)\right)=\mathfrak{I}\left(\omega\left(\mathcal{U}_{M}\right)\right)$ by Theorem 6.8. The two last equalities follow from Remark 6.9.

The next example shows that in general $\mathfrak{I}\left(\mathcal{U}_{M}\right) \neq \omega\left(\tau\left(\mathcal{U}_{M}\right)\right)$ and $\mathfrak{I}\left(\mathcal{U}_{M}^{H}\right) \neq$ $\omega\left(\tau\left(\mathcal{U}_{M}\right)\right)$ for a fuzzy metric space $(X, M, *)$.

Example 6.14 ([35]). Let $\left(M, *_{\mathrm{E}}\right)$ be the fuzzy metric on $X=(2,+\infty)$ given by

$$
M(x, y, t)= \begin{cases}1, & \text { if } x=y \\ \frac{1}{x}+\frac{1}{y}, & \text { if } x \neq y\end{cases}
$$

for all $x, y \in X$ and $t>0$ (hence $\left(M, *_{€}\right)$ is a stationary fuzzy metric since it does not depend on $t$ ).

It is easy to see that $\tau(M)$ is the discrete topology since $B_{M}(x, \varepsilon, t)=\{x\}$ whenever $0<\varepsilon<\frac{1}{2}-\frac{1}{x}$.

Now, let $a \in I^{X}$ be the characteristic function of the set $X \backslash\{3\}$. It is obvious that $a$ is continuous $\left(\tau(M)\right.$ is the discrete topology) so $a$ is $\omega\left(\tau\left(\mathcal{U}_{M}\right)\right.$ )-closed (in fact, $\omega\left(\tau\left(\mathcal{U}_{M}\right)\right)$ is the discrete topology). 
Nevertheless, let $\varepsilon \in I_{0}$ and $t>0$. We distinguish two cases:

(1) If $M(3, y, t)<1-\varepsilon$ for all $y \in X \backslash\{3\}$ then

$$
\bigvee_{y \in X} a(y) *_{\Xi}((1-\varepsilon) \rightarrow M(3, y, t))=\bigvee_{y \in X \backslash\{3\}}(M(3, y, t)+\varepsilon)=\frac{1}{3}+\frac{1}{2}+\varepsilon=\frac{5}{6}+\varepsilon .
$$

(2) If there exist $y_{0} \in X \backslash\{3\}$ such that $M\left(3, y_{0}, t\right) \geq 1-\varepsilon$ then

$$
\bigvee_{y \in X} a(y) *_{£}((1-\varepsilon) \rightarrow M(3, y, t)) \geq a\left(y_{0}\right) *_{\complement} 1=1
$$

Consequently

$$
\psi_{\mathfrak{I}\left(\mathcal{U}_{M}\right)}(a)(3)=\bigwedge_{\varepsilon \in I_{0}, t>0} \bigvee_{y \in X} a(y) *_{\mathrm{E}}((1-\varepsilon) \rightarrow M(3, y, t))=\frac{5}{6}>0=a(3) .
$$

Hence $\mathfrak{I}\left(\mathcal{U}_{M}\right) \neq \omega\left(\tau\left(\mathcal{U}_{M}\right)\right)$.

Similarly,

$$
\psi_{\mathfrak{I}\left(U_{M}^{H}\right)}(a)(3)=\bigwedge_{t>0} \bigvee_{y \in X} a(y) *_{\mathrm{E}} M(3, y, t)=\bigwedge_{t>0} \bigvee_{y \in X \backslash\{3\}} M(3, y, t)=\frac{5}{6}>0=a(3)
$$

and so $\mathfrak{I}\left(\mathcal{U}_{M}^{H}\right) \neq \omega\left(\tau\left(\mathcal{U}_{M}\right)\right)$.

Remark 6.15. The following diagrams summarize most results we have obtained:

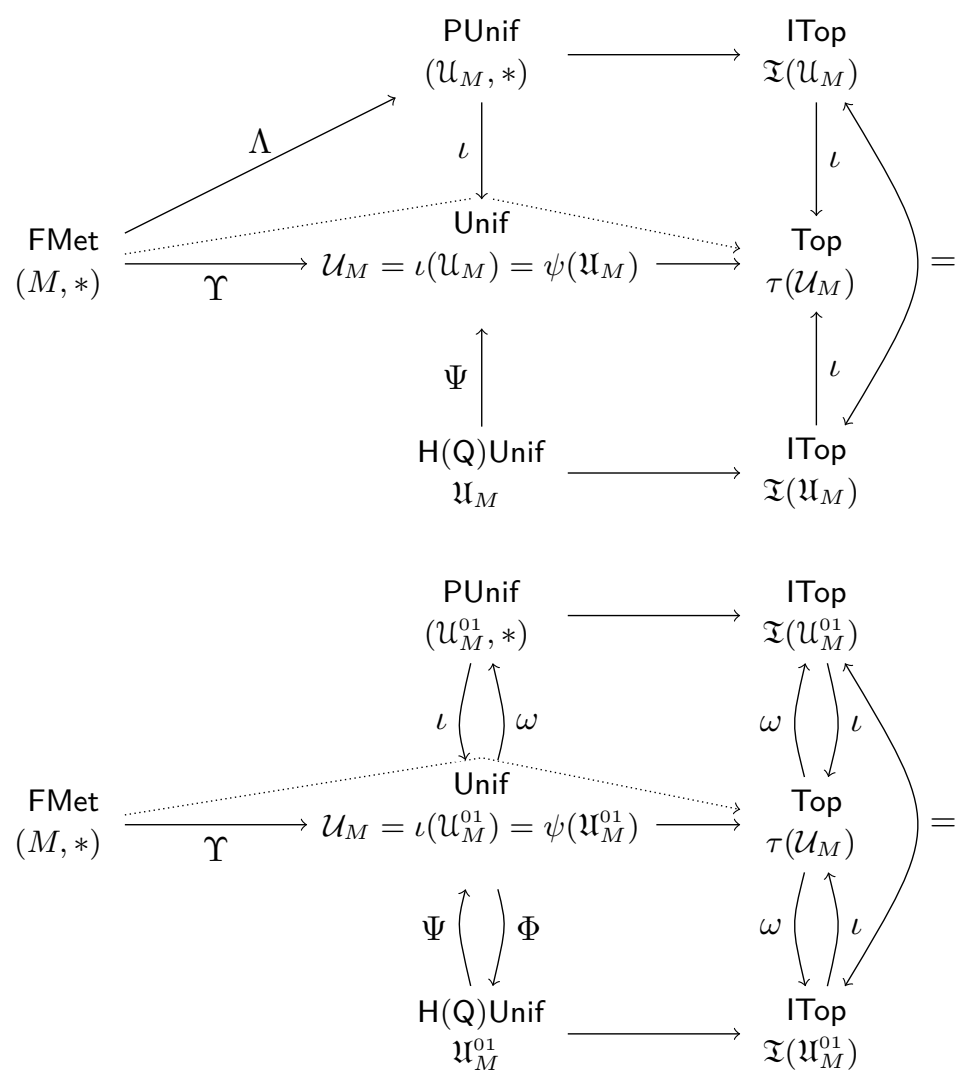




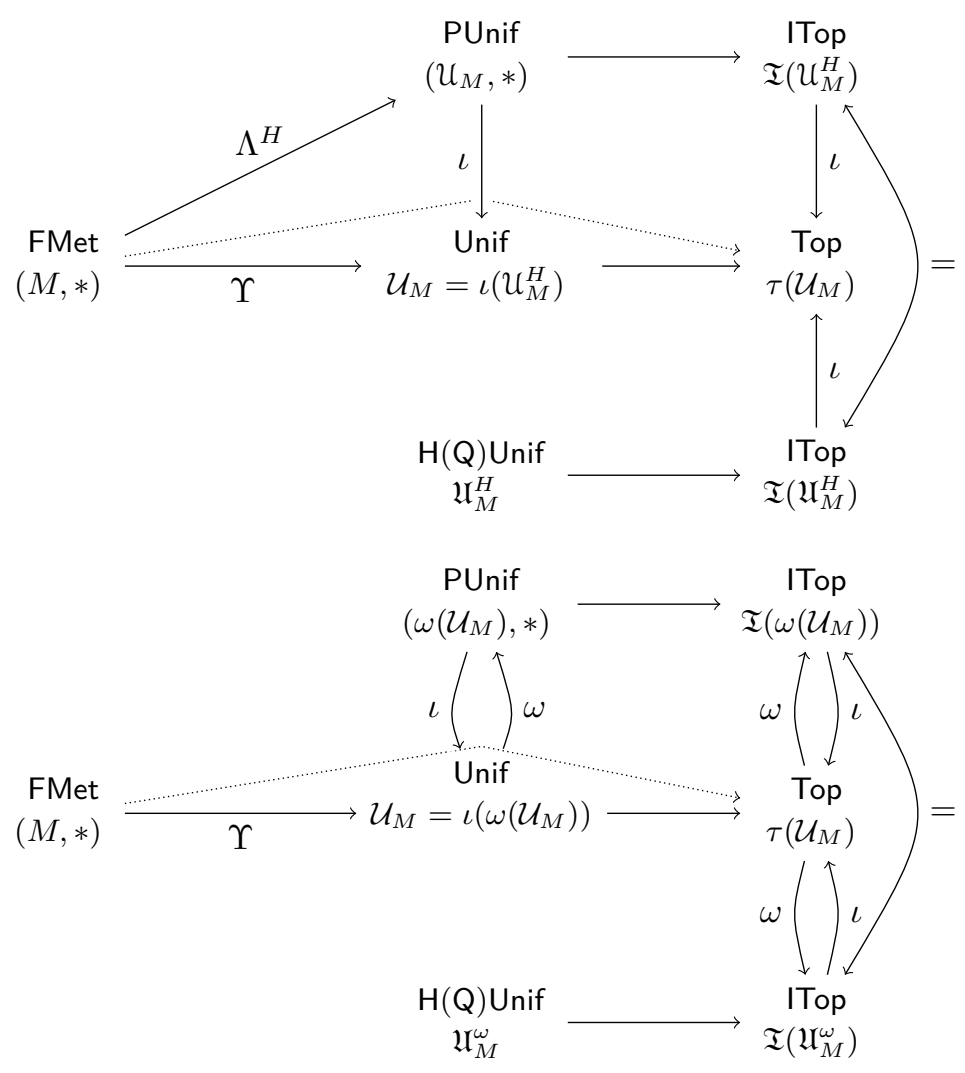

\section{ON FUZZY METRICS AND FUZZIFYING STRUCTURES}

Another approach to the study of topological spaces in the fuzzy context different from that of Chang and Lowen was due to Höhle [17], who introduced the concept of fuzzifying topology (see also [38]) under the name of $L$-fuzzy topology. His idea was to consider a fuzzy topology as a mapping T: $2^{X} \rightarrow I$ satisfying a multivalued version of the topological axioms (see Definition 7.9). On his behalf, Ying [39] used the same idea to introduce the concept of fuzzifying uniformity which was rediscovered in [2] under the name generalised uniformity. It was also proved in [2] that the category of fuzzifying uniform spaces is isomorphic to that of Lowen $\wedge$-uniform spaces.

On the other hand, Yue and Shi [40] developed a method to endow a fuzzy pseudometric space with a fuzzifying uniformity. Then it is natural to study the Lowen uniformity induced by this fuzzifying uniformity and obtain its relationship with the probabilistic uniformities that we have considered previously. To achieve this, we recall some pertinent concepts.

Given a nonempty set $X$, let $\mathcal{D}(X)$ denote the family of all $\varphi: 2^{X} \rightarrow 2^{X}$ such that:

- $A \subseteq \varphi(A)$ for all $A \in 2^{X}$;

- $\varphi\left(\cup_{i \in I} A_{i}\right)=\cup_{i \in I} \varphi\left(A_{i}\right)$ for all $\left\{A_{i} \mid I \in I\right\} \subseteq 2^{X}$ and $\varphi(\varnothing)=\varnothing$.

For $\varphi, \psi \in \mathcal{D}(X)$ we define $\varphi \preceq \psi$ if and only if $\varphi(A) \subseteq \psi(A)$ for each $A \in 2^{X}$. $\mathcal{D}(X)$ is a complete lattice in which the top and bottom elements $\varphi_{0}, \varphi_{1} \in \mathcal{D}(X)$ 
are given by

$$
\varphi_{0}(A)=A \quad \text { and } \quad \varphi_{1}(A)=\left\{\begin{array}{ll}
X, & \text { if } A \neq \varnothing ; \\
\varnothing, & \text { if } A=\varnothing ;
\end{array} \quad A \in 2^{X}\right.
$$

Since each $f \in \mathcal{D}(X)$ preserves arbitrary joins, it has a right adjoint map $\varphi^{\vdash}: 2^{X} \rightarrow 2^{X}$ given by

$$
\varphi^{\vdash}(B)=\bigcup\left\{A \in 2^{X} \mid \varphi(A) \subseteq B\right\}, \quad B \in 2^{X} .
$$

Since $\varphi^{\vdash}$ is meet preserving we can define $\varphi^{\triangleleft} \in \mathcal{D}(X)$ given by

$$
\varphi^{\triangleleft}(B)=X \backslash \varphi^{\vdash}(X \backslash B)=\bigcap\left\{C \in 2^{X} \mid \varphi(X \backslash C) \subseteq X \backslash B\right\}, \quad B \in 2^{X} .
$$

Definition 7.1 ([40], cf. $[39,30])$. A fuzzifying uniformity on a nonempty set $X$ is a mapping $\mathrm{U}: \mathcal{D}(X) \rightarrow I$ such that:

(FU1) $\mathrm{U}\left(\varphi_{1}\right)=1$;

(FU2) $\mathrm{U}(\varphi \wedge \psi)=\mathrm{U}(\varphi) \wedge \mathrm{U}(\psi)$ for all $\varphi, \psi \in \mathcal{D}(X)$;

(FU3) $\mathrm{U}(\varphi)=\bigvee_{\psi \circ \psi<\varphi} \mathrm{U}(\psi)$ for all $\varphi \in \mathcal{D}(X)$;

(FU4) $\mathrm{U}(\varphi)=\mathrm{U}\left(\varphi^{\triangleleft}\right)$ all $\varphi \in \mathcal{D}(X)$.

In this case, the pair $(X, \mathrm{U})$ is called a fuzzifying uniform space.

A function $f:(X, \mathrm{U}) \rightarrow(Y, \mathrm{~V})$ between two fuzzifying uniform spaces is said to be uniformly continuous if $\mathrm{V}(\varphi) \leq \mathrm{U}\left(f^{\Leftarrow}(\varphi)\right)$ for all $\varphi \in \mathcal{D}(Y)$, where $f^{\Leftarrow}(\varphi)(U)=$ $\{x \in X \mid f(x) \in \varphi(f(U))\}$ for all $U \in 2^{X}$.

We denote by FYUnif the category of fuzzifying uniform spaces and uniformly continuous functions.

In [2], the authors rediscovered fuzzifying uniformities under the name generalised uniformities. The definition of generalised uniformity differs formally from the definition of fuzzifying uniformity since a generalised uniformity is a function $d: 2^{X \times X} \rightarrow I$ satisfying certain properties instead of a function from $\mathcal{D}(X)$ to $I$ (we notice that generalised uniformities appear also in [30] under the name of fuzzifying uniformities). Nevertheless, it is well known that there is an order isomorphism between binary relations on $X$ and union preserving maps from $2^{X}$ to $2^{X}$. This isomorphism is given as follows:

$$
\begin{aligned}
U \in 2^{X \times X} \longmapsto \varphi_{U}: 2^{X} \rightarrow 2^{X}, \quad \varphi_{U}(A)=\{y \in X \mid(a, y) \in U \text { for some } a \in A\}, \\
\varphi: 2^{X} \rightarrow 2^{X} \longmapsto U_{\varphi}=\{(x, y) \in X \times X \mid y \in \varphi(\{x\})\} .
\end{aligned}
$$

Notice that if two union preserving maps $\varphi, \psi: 2^{X} \rightarrow 2^{X}$ coincide on the singletons then they coincide in the whole $2^{X}$. Moreover, given a binary relation $U$ on $X$, then $U$ is reflexive if and only if $\varphi_{U} \in \mathcal{D}(X)$. Consequently, each fuzzifying uniformity $\mathrm{U}: \mathcal{D}(X) \rightarrow I$ determines uniquely a generalised uniformity $d_{\mathrm{U}}: 2^{X \times X} \rightarrow I$ given by

$$
d_{\mathrm{U}}(U)=\left\{\begin{array}{ll}
\mathrm{U}\left(\varphi_{U}\right), & \text { if } \Delta \subseteq U ; \\
0, & \text { otherwise; }
\end{array} \quad U \in 2^{X \times X} .\right.
$$

Conversely, each generalised uniformity $d: 2^{X \times X} \rightarrow I$ determines uniquely a fuzzifying uniformity $\mathrm{U}_{d}: \mathcal{D}(X) \rightarrow I$ given by

$$
\mathrm{U}_{d}(\varphi)=d\left(U_{\varphi}\right) \quad \varphi \in \mathcal{D}(X) .
$$

In [2] the authors proved that the category of generalised uniform spaces is isomorphic to the category of Lowen $\wedge$-uniform spaces. To establish this result, let 
us recall [1] that if $(\mathcal{U}, *)$ is a probabilistic uniformity on a nonempty set $X$ then for all $\alpha \in I_{0}$,

$$
\mathcal{U}^{\alpha}=\left\{U^{\beta} \mid \beta<\alpha, U \in \mathcal{U}\right\}
$$

is a uniformity on $X$ called the $\alpha$-level uniformity of $\mathcal{U}$, where $U^{\beta}=\{(x, y) \in$ $X \times X \mid U(x, y)>\beta\}$. It is proved in [1] that a Lowen fuzzy uniformity is uniquely determined by its $\alpha$-level uniformities.

Remark 7.2. We notice that a similar reasoning can be applied to a fuzzy metric space $(X, M, *)$. In fact, given $\alpha \in I_{0}$ we can define a uniformity $\mathcal{U}_{M}^{\alpha}$, which we call the $\alpha$-level uniformity of $M$, having as base the family $\left\{U_{t}^{\beta} \mid 0<\beta<\alpha, t>0\right\}$, where $U_{t}^{\beta}=\{(x, y) \in X \times X \mid M(x, y, t)>\beta\}$. Then $\mathcal{U}_{M}=\bigvee_{\alpha \in I_{0}} \mathcal{U}_{M}^{\alpha}$ (cf. [30, Fact 1.6]).

Theorem $7.3([2])$. Let $(X, \mathrm{U})$ be a fuzzifying uniform space and $(X, \mathcal{U})$ be a $\wedge$ Lowen uniform space. Let us consider:

(1) the $\wedge$-Lowen uniformity $\mathcal{U}_{U}$ on $X$ given by

$$
\mathcal{U}_{U}=\left\{U \in I^{X \times X} \mid U^{\beta} \in \mathrm{U}^{\alpha} \text { for all } \alpha \in I_{0} \text { and } \beta<\alpha\right\}
$$

where $\mathrm{U}^{\alpha}=\left\{U \subseteq X \times X \mid \mathrm{U}\left(\varphi_{U}\right)>1-\alpha\right\}$ and $U^{\beta}=U^{-1}((\beta, 1])=\{(x, y) \in$ $X \times X \mid U(x, y)>\beta\}$

(2) the fuzzifying uniformity $\mathrm{U}_{u}: \mathcal{D}(X) \rightarrow I$ given by

$$
\mathrm{U}_{\mathcal{U}}(\varphi)= \begin{cases}1-\bigwedge S(\varphi), & \text { if } S(\varphi) \neq \varnothing ; \\ 0, & \text { if } S(\varphi)=\varnothing ;\end{cases}
$$

where $S(\varphi)=\left\{\alpha \in I \mid U_{\varphi} \in \mathcal{U}^{\alpha}\right\}$.

Then:

(i) the mapping $\mathfrak{s}$ : FYUnif $\rightarrow \operatorname{LUnif}(\wedge)$, which assigns to a fuzzifying uniformity $\mathrm{U}$ on $X$ the Lowen $\wedge$-uniformity $\mathcal{U}_{\cup}$ and which leaves morphisms unchanged, is a fully faithful functor;

(ii) the mapping $\mathfrak{f}$ : $\operatorname{LUnif}(\wedge) \rightarrow$ FYUnif, which assigns to a Lowen $\wedge$-uniformity $\mathfrak{U}$ the fuzzifying uniformity $\mathrm{U}_{\mathfrak{u}}$ and which leaves morphisms unchanged, is a fully faithful functor;

(iii) $\mathfrak{s} \circ \mathfrak{f}=1_{\text {FYUnif }}$ and $\mathfrak{f} \circ \mathfrak{s}=1_{\text {LUnif }(\wedge)}$.

On the other hand, in [40], the authors endowed a fuzzy pseudometric space with a fuzzifying uniformity as follows:

Theorem 7.4 ([40, Theorem 5.1]). Let $(M, \wedge)$ be a fuzzy pseudometric space. Then $\mathrm{U}_{M}^{F}: \mathcal{D}(X) \rightarrow I$ given by

$$
\mathrm{U}_{M}^{F}(\varphi)=\bigvee_{t>0} \bigwedge_{x \in X} \bigwedge_{y \notin \varphi(\{x\})}(1-M(x, y, t))
$$

is a fuzzifying uniformity on $X$.

Notice that Mardones-Pérez and de Prada Vicente gave a similar result in terms of generalised uniformities [30, Theorem 4.1]. Using the previous theorem, we intend to construct the $\wedge$-Lowen uniformity $\mathcal{U}_{M}^{F}:=\mathcal{U}_{\mathrm{U}_{M}^{F}}$ associated with the fuzzifying uniformity $\mathrm{U}_{M}^{F}$ of a fuzzy metric space as defined in Theorem 7.4. Let $(X, M, *)$ be a fuzzy metric space. 
Given $U \in I^{X \times X}$ we have that

$$
\begin{aligned}
U \in U_{M}^{F} & \Longleftrightarrow \forall \alpha \in I_{0}, \forall \beta<\alpha, U^{\beta} \in\left(\mathrm{U}_{M}^{F}\right)^{\alpha} \\
& \Longleftrightarrow \forall \alpha \in I_{0}, \forall \beta<\alpha, \mathrm{U}_{M}^{F}\left(\varphi_{U^{\beta}}\right)>1-\alpha \\
& \Longleftrightarrow \forall \alpha \in I_{0}, \forall \beta<\alpha, \bigvee_{t>0} \bigwedge_{x \in X} \bigwedge_{y \notin \varphi_{U^{\beta}}(\{x\})}(1-M(x, y, t))>1-\alpha \\
& \Longleftrightarrow \forall \alpha \in I_{0}, \forall \beta<\alpha, \bigvee_{t>0} \bigwedge_{x \in X} \bigwedge_{y \notin U^{\beta}(x)}(1-M(x, y, t))>1-\alpha \\
& \Longleftrightarrow \forall \alpha \in I_{0}, \forall \beta<\alpha, \bigwedge_{t>0} \bigvee_{x \in X} \bigvee_{y \notin U^{\beta}(x)} M(x, y, t)<\alpha \\
& \Longleftrightarrow \forall \alpha \in I_{0}, \forall \beta<\alpha, \exists s>0: \bigvee_{x \in X} \bigvee_{y \notin U^{\beta}(x)} M(x, y, s)<\alpha \\
& \Longleftrightarrow \forall \alpha \in I_{0}, \forall \beta<\alpha, \exists s>0, \exists \alpha^{\prime}<\alpha: \bigvee_{x \in X} \bigvee_{y \notin U^{\beta}(x)} M(x, y, s) \leq \alpha^{\prime} \\
& \Longleftrightarrow \forall \alpha \in I_{0}, \forall \beta<\alpha, \exists s>0, \exists \alpha^{\prime}<\alpha: U_{M, s}^{\alpha^{\prime}} \subseteq U^{\beta} \\
& \Longleftrightarrow \forall \alpha \in I_{0}, \forall \beta<\alpha, U^{\beta} \in \mathcal{U}_{M}^{\alpha} .
\end{aligned}
$$

Hence

$$
\mathcal{U}_{M}^{F}=\left\{U \in I^{X \times X} \mid U^{-1}((\beta, 1]) \in \mathcal{U}_{M}^{\alpha} \text { for all } \alpha \in I_{0} \text { and } \beta<\alpha\right\} .
$$

Proposition 7.5. Let $(X, M, *)$ be a fuzzy metric space. Then:

$$
\mathcal{U}_{M}^{H} \subseteq \mathcal{U}_{M}^{F} \subseteq \omega\left(\mathcal{U}_{M}\right)
$$

Proof. Let $\alpha \in I_{0}$ and $t>0$. Given $\beta \in I$ with $\beta<\alpha$ then $M_{t}^{-1}((\beta, 1])=$ $\{(x, y) \in X \times X \mid M(x, y, t)>\beta\} \in \mathcal{U}_{M}^{\alpha}$ so $M_{t} \in \mathcal{U}_{M}^{F}$. Hence we deduce the first inclusion. The other inclusion is obvious by definition of $\omega\left(\mathcal{U}_{M}\right)$ since $\mathcal{U}_{M}^{\alpha} \subseteq \mathcal{U}_{M}$ for all $\alpha \in I_{0}$.

We provide two examples showing that the above inclusions are, in general, strict.

Example 7.6. Let us consider the fuzzy metric space $(\mathbb{R}, M, \cdot)$ where $M$ is given by

$$
M(x, y, t)=\left\{\begin{array}{ll}
1, & \text { if } x=y \\
\frac{1}{2}, & \text { if } x \neq y
\end{array} .\right.
$$

Then it is obvious that $\mathcal{U}_{M}=\mathcal{U}_{M}^{\alpha}$ is the discrete uniformity for all $\alpha \in I_{0}$. Let $F \in I^{\mathbb{R} \times \mathbb{R}}$ be given by

$$
F(x, y)=\left\{\begin{array}{ll}
1 & \text { if } x=y \\
\frac{1}{3} & \text { if } x \neq y
\end{array} .\right.
$$

Clearly $F^{-1}((\varepsilon, 1]) \in U_{M}^{\alpha}$ for all $\varepsilon, \alpha \in I_{1}$ so $F \in \mathcal{U}_{M}^{F}$. Nevertheless, $F \notin \mathcal{U}_{M}^{H}$ since if $x \neq y$ then $M(x, y, t)>F(x, y)$ so $M_{t} \not \leq F$ for all $t>0$. Consequently, $\mathcal{U}_{M}^{H} \neq \mathcal{U}_{M}^{F}$.

Example 7.7. Let us consider the real line $\mathbb{R}$ endowed with the euclidean metric $e$. Let us define the fuzzy metric $(N, \cdot)$ on $\mathbb{R}$ given by

$$
N(x, y, t)=\max \left\{M_{e}(x, y, t), \frac{1}{2}\right\},
$$

where $M_{e}$ is the standard fuzzy metric associated with the euclidean metric. Then $U=\left\{(x, y) \in \mathbb{R} \times \mathbb{R} \mid e(x, y)<\frac{1}{2}\right\} \in \mathcal{U}_{e}=\mathcal{U}_{M_{e}}=\mathcal{U}_{N}$ and so $1_{U} \in \omega\left(\mathcal{U}_{N}\right)$. Given $0<\varepsilon<\frac{1}{2}$ and $t>0$ then $N(x, y, t)>\varepsilon$ for all $x, y \in X$ so $U_{t}^{\varepsilon}=\{(x, y) \in X \times X \mid$ 
$N(x, y, t)>\varepsilon\}=X \times X$. Hence $\mathcal{U}_{N}^{1 / 2}=\{X \times X\}$ so $1_{U}^{-1}((\varepsilon, 1])=U \notin \mathcal{U}_{N}^{1 / 2}$ for all $0<\varepsilon<\frac{1}{2}$. Consequently $1_{U} \notin \mathcal{U}_{N}^{F}$, i.e. $\omega\left(\mathcal{U}_{N}\right) \nsubseteq \mathcal{U}_{N}^{F}$.

This also shows that $\mathcal{U}_{N}^{01} \nsubseteq \mathcal{U}_{N}^{F}$.

Nevertheless, we can prove the following:

Proposition 7.8. Let $(X, d)$ be a metric space. Then

$$
\mathcal{U}_{M_{d}}^{F}=\omega\left(\mathcal{U}_{d}\right) \text {. }
$$

Proof. We only need to prove that $\omega\left(\mathcal{U}_{d}\right) \subseteq \mathcal{U}_{M_{d}}^{F}$. Let us suppose that $U \in \omega\left(\mathcal{U}_{d}\right)$. Given $\alpha \in I_{0}$ and $0<\varepsilon<\alpha$ we have that $U^{-1}((\varepsilon, 1]) \in \mathcal{U}_{d}=\mathcal{U}_{M_{d}}$ so we can find $\gamma \in I_{1}$ and $t>0$ such that $M_{d}(x, y, t)>\gamma$ implies $U(x, y)>\varepsilon$. If $\gamma<\alpha$ we immediately have that $U^{-1}((\varepsilon, 1]) \in \mathcal{U}_{M_{d}}^{\alpha}$. Otherwise, fix $0<\beta<\alpha$. Since $\lim _{s \rightarrow 0^{+}} s(1-\beta) / \beta=0$ we can find $s_{0}>0$ such that $t(1-\gamma) / \gamma>s_{0}(1-\beta) / \beta$. An easy computation shows that if $M_{d}\left(x, y, s_{0}\right)>\beta$ then $M_{d}(x, y, t)>\gamma$ and so $U(x, y)>\varepsilon$. Therefore, $U^{-1}((\varepsilon, 1]) \in \mathcal{U}_{M_{d}}^{\alpha}$ which finishes the proof.

Finally we will study in what follows the relation between fuzzy metrics and fuzzifying topologies. Recall that Yue and Shi [40] endowed a fuzzy metric space with a fuzzifying topology. Note also that Zhang and $\mathrm{Xu}$ [44] proved that the category of fuzzifying topological spaces is isomorphic to that of fuzzy neighborhood spaces, a special kind of stratified $I$-topological spaces [37]. In the following we study the $I$-topology associated to a fuzzy metric space $(X, M, *)$ constructed by means of its fuzzifying topology. In particular, we study its relationship with the $I$-topology $\omega(\tau(M))$ that we have previously considered. We begin by recalling some pertinent definitions.

Definition 7.9 ([17, 38]). A fuzzifying topology on a nonempty set $X$ is a function $\mathrm{T}: 2^{X} \rightarrow I$ such that:

(FY1) $\mathrm{T}(X)=\mathrm{T}(\varnothing)=1$;

(FY2) $\mathrm{T}(U \cap V) \geq \mathrm{T}(U) \wedge \mathrm{T}(V)$;

(FY3) $\mathrm{T}\left(\cup_{i \in \Lambda} U_{i}\right) \geq \bigwedge_{i \in \Lambda} \mathrm{T}\left(U_{i}\right)$.

In this case, the pair $(X, \mathrm{~T})$ is called a fuzzifying topological space.

A function $f:\left(X, \mathrm{~T}_{X}\right) \rightarrow\left(Y, \mathrm{~T}_{Y}\right)$ between two fuzzifying topological spaces is said to be continuous if $\mathrm{T}_{Y}(U) \leq \mathrm{T}_{X}\left(f^{-1}(U)\right)$ for each $U \subseteq Y$.

We denote by FYS the category of fuzzifying topological spaces and continuous functions.

Yue and Shi [40] constructed a fuzzifying topology from a fuzzy metric space as follows:

Proposition $7.10([40])$. Let $(X, M, *)$ be a fuzzy pseudometric space and for each $x \in X$ define $\mathrm{T}_{M}: 2^{X} \rightarrow I$ as:

$$
\mathrm{T}_{M}(U)=\bigwedge_{x \in U} \bigvee_{t>0} \bigwedge_{y \notin U}(1-M(x, y, t)), \quad U \subseteq X .
$$

Then $\mathrm{T}_{M}$ is a fuzziying topology on $X$.

Definition 7.11 ([28]). A fuzzy neighborhood system on a set $X$ is a family of prefilters $(\mathcal{U}(x))_{x \in X}$ satisfying:

(FN1) $a(x)=1$ for all $a \in \mathcal{U}(x)$;

(FN2) $\mathcal{U}(x)$ is saturated for all $x \in X$; 
(FN3) given $x \in X, a \in \mathcal{U}(x)$ and $\varepsilon>0$ there exists a family $\left\{a_{y}^{\varepsilon} \in \mathcal{U}(y) \mid y \in X\right\}$ such that $a_{x}^{\varepsilon}(z) \wedge a_{z}^{\varepsilon}(y) \leq a(y)+\varepsilon$ for all $z, y \in X$.

In this case the pair $\left(X,(\mathcal{U}(x))_{x \in X}\right)$ is called a fuzzy neighborhood space.

On the other hand, a continuous map between two fuzzy neighborhood spaces $\left(X,(\mathcal{U}(x))_{x \in X}\right)$ and $\left(Y,(\mathcal{V}(y))_{y \in Y}\right)$ is a function $f: X \rightarrow Y$ such that $a \circ f \in \mathcal{U}(x)$ for all $x \in X$ and all $a \in \mathcal{V}(f(x))$. We will denote by FNS the category of fuzzy neighborhood spaces and continuous maps.

Furthermore, if $(\mathcal{U}(x))_{x \in X}$ is a fuzzy neighborhood system on a nonempty set $X$ then it induces a stratified fuzzy topology $\mathfrak{I}\left((\mathcal{U}(x))_{x \in X}\right)$ on $X$ whose closure operator is given by

$$
\bar{a}(x)=\bigwedge_{b \in \mathcal{U}(x)} \bigvee_{y \in X} a(y) \wedge b(y)
$$

where $a \in I^{X}$ and $x \in X$. Wuyts, Lowen and Lowen [37] characterized those stratified fuzzy topological spaces which have a compatible fuzzy neighborhood system. Later on, Zhang and Xu [44] proved that the category FYS is isomorphic to the category FNS:

Theorem 7.12 ([44]). Let $\mathrm{T}$ be a fuzzifying topology on $X$ and $\mathfrak{I}$ a stratified fuzzy topology on $X$. Let us consider:

(1) the stratified fuzzy topology $\mathfrak{I}_{\mathrm{T}}$ on $X$ given by

$$
\mathfrak{I}_{\mathrm{T}}=\left\{a \in I^{X} \mid \forall \alpha \in I_{1}, a^{-1}((\alpha, 1]) \in \iota_{\alpha}(\mathrm{T})\right\}
$$

where $\iota_{\alpha}(\mathrm{T})=\bigvee_{\beta>\alpha}\{U \subseteq X \mid \mathrm{T}(U) \geq \beta\}$

(2) the fuzzifying topology $\mathrm{T}_{\mathfrak{I}}$ on $X$ given by

$$
\mathrm{T}_{\mathfrak{I}}(U)=\bigvee\left\{\alpha \in I_{1} \mid U=a^{-1}((\alpha, 1]) \text { for some } a \in \mathfrak{I}\right\} .
$$

Then:

(i) the mapping $\mathfrak{s}: \mathrm{FYS} \rightarrow$ FNS, which assigns to a fuzzifying topology $\mathrm{T}$ on $X$ the fuzzy neighborhood system compatible with $\mathfrak{I}_{\mathrm{T}}$ and which leaves morphisms unchanged, is a fully faithful functor;

(ii) the mapping $\mathfrak{\mathfrak { f }}:$ FNS $\rightarrow$ FYS, which assigns to a fuzzy neighborhood system $(\mathcal{U}(x))_{x \in X}$ on $X$ the fuzzifying topology $\mathrm{T}_{\mathfrak{I}\left((\mathcal{U}(x))_{x \in X}\right)}$ associated with the stratified fuzzy topology $\mathfrak{I}\left((\mathcal{U}(x))_{x \in X}\right)$ and which leaves morphisms unchanged, is a fully faithful functor;

(iii) $\mathfrak{s} \circ \mathfrak{f}=1_{\mathrm{FYS}}$ and $\mathfrak{f} \circ \mathfrak{s}=1_{\mathrm{FNS}}$.

Using the above results, we can construct from a fuzzy metric space $(X, M, *)$ a stratified fuzzy topology $\mathfrak{I}_{T_{M}}$ associated with the fuzzifying topology generated by a fuzzy pseudometric as defined in Proposition 7.10. Then we have:

Proposition 7.13. Let $(X, M, *)$ be a fuzzy metric space. Then

$$
\mathfrak{I}_{\mathrm{T}_{M}} \subseteq \omega(\tau(M)) \text {. }
$$

Proof. Let $a \in \mathfrak{I}_{\mathrm{T}_{M}}$ and $\varepsilon \in I_{1}$. Given $x_{0} \in a^{-1}((\varepsilon, 1])$ we can find $U \subseteq X$ with $x_{0} \in U \subseteq a^{-1}((\varepsilon, 1])$ and $\mathrm{T}_{M}(U)=\bigwedge_{x \in U} \bigvee_{t>0} \bigwedge_{y \notin U}(1-M(x, y, t)) \geq \beta>\varepsilon$ for some $\varepsilon<\beta<1$. Let $t_{0}>0$ such that $\bigwedge_{y \notin U}\left(1-M\left(x_{0}, y, t_{0}\right)\right)>\varepsilon$. It is easy to see that $B_{M}\left(x_{0}, \varepsilon, t_{0}\right) \subseteq U \subseteq a^{-1}((\varepsilon, 1])$. In fact, given $y \in B_{M}\left(x_{0}, \varepsilon, t_{0}\right)$ if $y \notin U$ then $1-M\left(x_{0}, y, t_{0}\right)>\varepsilon$, i.e. $M\left(x_{0}, y, t_{0}\right)<1-\varepsilon$ a contradiction. Hence $a^{-1}((\varepsilon, 1]) \in \tau(M)$ for all $\varepsilon \in I_{1}$ so $a \in \omega(\tau(M))$. 
The following example shows that the above inclusion is, in general, strict.

Example 7.14. Let us consider the fuzzy metric $(N, \cdot)$ as defined in Example 7.7. It is clear that the function $a(x)=\frac{x^{2}+1}{x^{2}+2}$ belongs to $\omega(\tau(N))$ since it is a continuous function. Nevertheless, we next show that $a \notin \mathfrak{I}_{\mathrm{T}_{N}}$. Let us consider $V=a^{-1}\left(\left(\frac{3}{4}, 1\right]\right)=(-\infty,-\sqrt{2}) \cup(\sqrt{2}, \infty)$. Since $\frac{1}{2} \leq N(x, y, t) \leq 1$ for every $x, y \in X$ and $t>0$ then $0 \leq \mathrm{T}_{N}(U) \leq \frac{1}{2}$ for every $U \subseteq X$. Hence $V \notin \iota_{\frac{3}{4}}\left(\mathrm{~T}_{N}\right)$.

\section{ACKNOWLEDGEMENTS}

The authors are very grateful to the referees and the area editor for their suggestions which have improved the first version of the paper.

\section{REFERENCES}

[1] M.H. Burton, The relationship between a fuzzy uniformity and its family of $\alpha$-level uniformities, Fuzzy Sets Syst. 54 (1993), 311-315.

[2] M.H. Burton, M.A. de Prada Vicente, and J. Gutiérrez García, Generalised uniform spaces, J. Fuzzy Math. 4 (1996), 363-380.

[3] C.L. Chang, Fuzzy topological spaces, J. Math. Anal. Appl. 24 (1968), 182-190.

[4] R. Engelking, General topology, vol. 6 of Sigma Series in Pure Mathematics, Heldermann Verlag Berlin, 1989.

[5] P. Fletcher and W.F. Lindgren, Quasi-Uniform Spaces, Marcel Dekker, New York, 1982.

[6] A. George and P. Veeramani, On some results in fuzzy metric spaces, Fuzzy Sets Syst. 64 (1994), no. 3, 395-399.

[7] A. George and P. Veeramani, Some theorems in fuzzy metric spaces, J. Fuzzy Math. 3 (1995), no. 4, 933-940.

[8] M. Grabiec, Fixed points in fuzzy metric spaces, Fuzzy Sets Syst. 27 (1988), no. 3, 385-389.

[9] V. Gregori and S. Romaguera, Some properties of fuzzy metric spaces, Fuzzy Sets Syst. 115 (2000), no. 3, 485-489.

[10] V. Gregori and S. Romaguera, On completion of fuzzy metric spaces, Fuzzy Sets Syst. 130 (2002), no. 3, 399-404.

[11] V. Gregori and S. Romaguera, On fuzzy quasi-metric spaces, Appl. Gen. Topol. 5 (2004), no. $1,129-136$.

[12] J. Gutiérrez García and M.A. de Prada Vicente, Hutton [0,1]-quasi-uniformities induced by fuzzy (quasi-)metric spaces, Fuzzy Sets Syst. 157 (2006), no. 6, 755-766.

[13] J. Gutiérrez García, M.A. de Prada Vicente, and S. Romaguera, Completeness of Hutton [0,1]-quasi-uniform spaces, Fuzzy Sets Syst. 158 (2007), no. 16, 1791-1802.

[14] J. Gutiérrez García, M.A. de Prada Vicente, and A.P. Šostak, A unified approach to the concept of fuzzy L-uniform space, Topological and algebraic structures in fuzzy sets (S. E. Rodabaugh and E. P. Klement, eds.), Kluwer Academic Publishers, 2003, pp. 81-114.

[15] J. Gutiérrez García, S. Romaguera, and M. Sanchis, Fuzzy uniform structures and continuous t-norms, Fuzzy Sets Syst. 161 (2010), no. 7, 1011-1021.

[16] U. Höhle, Probabilistic uniformization of fuzzy topologies, Fuzzy Sets Syst. 1 (1978), 311-332.

[17] U. Höhle, Upper semicontinuous fuzzy sets and applications, J. Math. Anal. Appl. 78 (1980), 659-673

[18] U. Höhle, Probabilistic topologies induced by L-fuzzy uniformities, Manuscripta Math. 38 (1982), 289-323.

[19] U. Höhle, Probabilistic metrization of fuzzy uniformities, Fuzzy Sets Syst. 8 (1982), 63-69.

[20] B. Hutton, Uniformities on fuzzy topological spaces, J. Math. Anal. Appl. 58 (1977), 559-571.

[21] A. Katsaras, Fuzzy proximity spaces, J. Math. Anal. Appl. 68 (1979), 100-110.

[22] A. Katsaras, On fuzzy uniform spaces, J. Math. Anal. Appl. 101 (1984), 97-113.

[23] A. Katsaras, Linear fuzzy neighborhood spaces, II, J. Math. Anal. Appl. 115 (1986), 560-573.

[24] I. Kramosil and J. Michalek, Fuzzy metrics and statistical metric spaces, Kybernetica 11 (1975), no. 5, 336-344.

[25] R. Lowen, Fuzzy topological spaces and fuzzy compactness, J. Math. Anal. Appl. 56 (1976), no. 3, 621-633. 
[26] R. Lowen, Convergence in fuzzy topological spaces, General Topology Appl. 10 (1979), 147160.

[27] R. Lowen, Fuzzy uniform spaces, J. Math. Anal. Appl. 82 (1981), no. 2, 370-385.

[28] R. Lowen, Fuzzy neighbourhood spaces, Fuzzy Sets Syst. 7 (1982), 165-189.

[29] R. Lowen, Completeness, compactness and precompactness in fuzzy uniform spaces: part I, J. Math. Anal. Appl. 90 (1982), no. 2, 563-581.

[30] I. Mardones-Pérez and M.A. de Prada Vicente, Fuzzy pseudometric spaces vs fuzzifying structures, Fuzzy Sets Syst. 267 (2015), 117-132.

[31] K. Menger, Statistical metrics, Proc. Nat. Acad. Sci. U.S.A. 28 (1942), 535-?537

[32] S.E. Rodabaugh, A theory of fuzzy uniformities with applications to the fuzzy real lines, J. Math. Anal. Appl. 129 (1988), 37-70.

[33] J. Rodríguez-López, Fuzzy uniform structures, Filomat, to appear.

[34] J. Rodríguez-López and S. Romaguera, The Hausdorff fuzzy metric on compact sets, Fuzzy Sets Syst. 147 (2004), 273-283.

[35] A. Sapena, A contribution to the study of fuzzy metric spaces, Appl. Gen. Topol. 2 (2001), no. $1,63-75$.

[36] B. Schweizer and A. Sklar, Statistical metric spaces, Pacific J. Math. 10 (1960), 314-334.

[37] P. Wuyts, R. Lowen, and E. Lowen, Reflectors and coreflectors in the category of fuzzy topological spaces, Comput. Math. Applic. 16 (1988), 823-836.

[38] M.S. Ying, A new approach for fuzzy topology (I), Fuzzy Sets Syst. 39 (1991), 303-321.

[39] M.S. Ying, Fuzzifying uniform spaces, Fuzzy Sets Syst. 53 (1993), 93-104.

[40] Y. Yue and F.-G. Shi, On fuzzy pseudo-metric spaces, Fuzzy Sets Syst. 161 (2010), 11051116.

[41] Y. Yue and J. Fang, Uniformities in fuzzy metric spaces, Iranian J. Fuzzy Syst. 12, no. 1 (2015), 43-57.

[42] D. Zhang, A comparison of various uniformities in fuzzy topology, Fuzzy Sets Syst. 140 (2003), no. 3, 399-422.

[43] D. Zhang, Uniform environments as a general framework for metrics and uniformities, Fuzzy Sets Syst. 159 (2008), no. 3, 559-572.

[44] D. Zhang and L. Xu, Categories isomorphic to FNS, Fuzzy Sets Syst. 104 (1999), 373-380.

(J. Gutiérrez García) Department of Mathematics, University of the Basque Country UPV/EHU, Apdo. 644, 48080 BilbaO, Spain

E-mail address: javier.gutierrezgarcia@ehu.eus

(J. Rodríguez-López) Instituto Universitario de Matemática Pura y Aplicada, UniverSitat Politècnica de València, Camino de Vera s/n, 46022 Valencia, Spain

E-mail address: jrlopez@mat.upv.es

(S. Romaguera) Instituto Universitario de Matemática Pura y Aplicada, Universitat Politècnica de València, Camino de Vera s/N, 46022 Valencia, Spain

E-mail address: sromague@mat.upv.es 